\title{
Stefan Meyer und die Radioaktivitätsforschung in Österreich
}

Die frühe Geschichte der Erforschung der Radioaktivität in Österreich und ihre weitere Entwicklung sind untrennbar mit dem wissenschaftlichen Lebensweg des Physikers Stefan Meyer verknüpft. Seit der Zeit der ersten Entdekkungen auf diesem für die Physik des 20. Jahrhunderts revolutionären Gebiet der Radioaktivität bis zur Institutionalisierung der Radioaktivitätsforschung am Wiener Institut für Radiumforschung ist Meyer die prägende Autorität dieser neuen Forschungsrichtung weit über die Grenzen Österreichs hinaus. ${ }^{1}$

Die vorliegende Arbeit schließt die Absicht mit ein, weiterführende wissenschaftshistorische Auseinandersetzungen mit den Forschungsleistungen österreichischer Wissenschaftler auf dem Gebiet der Radioaktivität anzuregen und die Aufmerksamkeit auf die Rolle des Wiener Radiuminstituts und der dort tätigen Forscher bei der Etablierung und Institutionalisierung der Radioaktivitätsforschung zu lenken. Das im Anhang hier erstmals präsentierte Verzeichnis der Schriften von Stefan Meyer schafft dafür eine der notwendigen Voraussetzungen.

\section{Meyer - FAMilie und LehrJahre}

Stefan Julius Meyer wurde am 27. April 1872 in Wien als zweiter Sohn des Dr. Gotthelf Karl Meyer (1844-1905) und dessen Gattin Clara, geborene Goldschmidt (1847-1924) geboren. Sein Vater war Jurist und Notar zunächst in Horn, Niederösterreich, dann in Wien und neben seinem Beruf auch literarisch tätig (er verfaßte mehrere Bände des „Neuen Pitaval“) und führte nebenher auch einen Verlag und eine Buchhandlung. Dem Wiener Musikleben ist Stefan Meyers Vater, der für den häuslichen Gebrauch selbst auf der Violine dilettiert, als Mitglied des Direktoriums der Wiener „Gesellschaft der Musikfreunde“ eng verbunden und er ist zudem Gründungsmitglied der „Wiener Brahms-Gesellschaft", die den verehrten Komponisten auch als Gast in die Wohnung in der damaligen Reichsratstraße 5 führt. Meyers Mutter war die Schwester des bekannten Mineralogen und Petrographen an der Universität Heidelberg, Viktor M. Goldschmidt (1853-1933), und entstammte einer Mainzer Familie, die nach 1866 Mainz verließ, um sich in England und Österreich anzusiedeln.

Die Orte, wo Meyers Vorfahren lebten und arbeiteten, spannen eine weite europäische Landkarte auf: Der bedeutende Arzt, Chemiker und Naturforscher Josef Manes Österreicher der väterlichen Linie wird 1755 in Altofen geboren, bekleidet in Wien unter Joseph II., Leopold II. und Franz II. die Funktion eines Hof- und Staatsarzts (Kameralphysicus) und stirbt hier, viel-

${ }^{1}$ Die vorliegende Arbeit ist eine erweiterte Fassung des Vortrags anläßlich der Veranstaltung „Die Erforschung der Radioaktivität in Österreich - Stefan Meyer zum 50. Todestag“ der Kommission für Geschichte der Naturwissenschaften, Mathematik und Medizin der Österreichischen Akademie der Wissenschaften am 6. Mai 2000. 




Abb. 1: Stefan Meyer als Lieutenant in der Reserve des 14. Corps-Artillerie Regiments (1892). Archiv der Österreichischen Zentralbibliothek für Physik.

fach ausgezeichnet, 1831. Die Vorfahren seines Vaters Gotthelf Karl, Levin Jehuda Meyer (1770-1844) und dessen Sohn Isaak Lewin Meyer (1802-1871), der 1842 in Wien eine Import-Exportfirma eröffnet, kommen aus dem norddeutschen Raum um Stavenhagen und Hamburg mit Verbindungen zur Familie des Dichters Heinrich Heine. Die Mutter Stefan Meyers, Clara Regina Goldschmidt, deren Eltern aus alten Frankfurter und Prager Familien stammen, wird 1847 in Mainz geboren. Der Verbindung des Berliner Rechtsanwalts Felix Maass (1852-1920) mit Fanny von Portheim (1860-1949) aus Prag entstammt Meyers Frau Emmy, geboren 1884 in Berlin. Stefan Meyers Schwester Herta (1876-1958) ist mit dem Vulkanologen Immanuel Friedländer (1871-1948) verheiratet, der unter anderem in Neapel sein eigenes vulkanologisches Forschungsinstitut unterhält. Das älteste der vier Kinder des Ehepaars Meyer, Hans Meyer (1871-1942), wirkt als Professor für organische Chemie an der Deutschen Universität in Prag. Verwandte leben als Geschäftsleute in England; eine europäische Familie des 19. Jahrhunderts, die Kaufleute, Industrielle, Ärzte und Wissenschaftler hervorgebrachte.

Mit Absicht und Bedacht binde ich meine Reflexionen zu Stefan Meyer in die Geschichte der Juden in Österreich, die Geschichte der österreichischen Juden, ein. Und dies deshalb, weil die Person Meyers nur vor der Folie seiner jüdischen und europäischen Familie zutreffend beschreibbar und biographisch begreifbar wird. Dieser Kontext mag Meyer selbst fremd gewesen sein, wir wissen es nicht, wir können dies nur vermuten aufgrund der Tatsache seiner Herkunft aus dem liberalen österreichischen Bürgertum, für welches für 


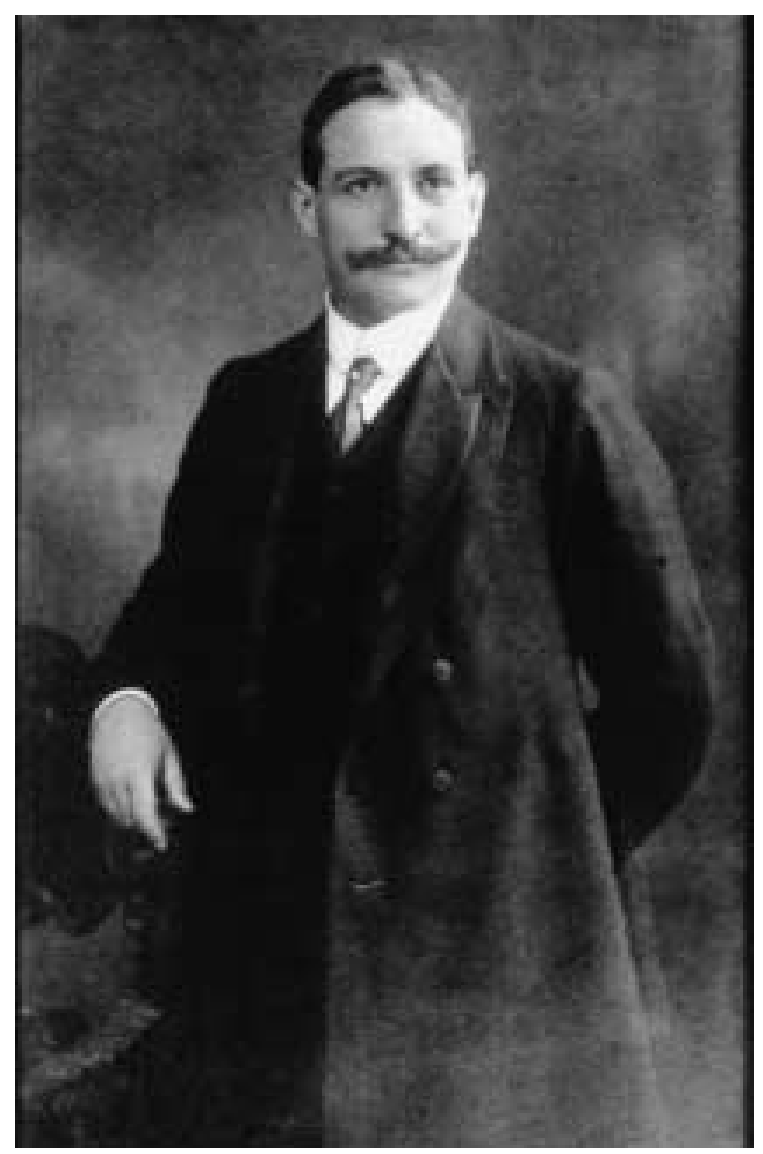

Abb. 2: Stefan Meyer (ca. 1908). Archiv der Österreichischen Zentralbibliothek für Physik.

eine kurze Spanne seiner Geschichte, rassische und religiöse Zuordnungen, wie sie in vernichtender Absicht das nationalsozialistische Regime herstellte, fremd gewesen sein mögen.

Hier mag die Frage berechtigt erscheinen, inwieferne dieser Kontext für Meyer als Wissenschafter relevant ist. Die folgenden Aspekte seien hier erläuternd erwähnt: Der familiäre Hintergrund der Vorfahren Meyers verweist zurück auf die Zeit der Aufklärung und die beginnende Emanzipation der Juden in Europa und in Österreich. Und insofern ist die Familienherkunft gerade auch eines Wissenschaftlers mit den sozialen und ökonomischen Entwicklungen, dem Fortschrittsglauben der liberalen jüdischen Bourgeoisie und deren Vertrauen in die Rationalität der Wissenschaft eng verbunden mit Karriereverläufen, für die auch jener Stefan Meyers steht.

Meyer studierte nach Volksschuljahren in Wien und nach Absolvierung des Gymnasiums in Horn an der Universität Wien und im Wintersemester 1894/95 in Leipzig Physik, Mathematik und Chemie und promovierte 1896 bei Franz Serafin Exner (1849-1926)² mit der Arbeit „Über den Sitz der Potentialdifferenzen in Tropfelektroden und im Capillarelektrometer"3. Meyers

2 Berta Karlik und Erich Schmid, „Franz Serafin Exner und sein Kreis. Ein Beitrag zur Geschichte der Physik in Österreich“ (Wien: Österr. Akad. d. Wiss., 1982).

3 Stefan Meyer, „Über den Sitz der Potentialdifferenzen in Tropfelektroden und im Capillarelektrometer," Sitzungsberichte der kaiserl. Akad. d. Wiss., mathem.-naturw. Kl., Bd. CV, Abt. IIa, Jänner 1896, 136-160. 
Beschäftigung mit Musik - er selbst spielte die Baßgeige - ging über die Pflege der Hausmusik, an der er in der Familie Exner regen Anteil nahm, hinaus. Sein lebenslanges Interesse für Probleme der Akustik und der Instrumentenkunde ist in seiner Dozententätigkeit am Wiener Konservatorium in den Jahren 1902 bis 1911 begründet. Und noch in der Zeit als „Zwangspensionist" - wie Meyer selbst sein Dasein in den Jahren von 1938 bis 1945 kühl und ironisch distanziert charakterisiert - verfasste er zusammen mit dem Philharmoniker Alexander Wunderer die posthum veröffentlichten „Grundlagen der Instrumentenkunde für Musikanten und Dilettanten“. 4

Stefan Meyer erfuhr das für einen Wissenschaftler seltene Glück, Pionierarbeit auf wissenschaftlichem Neuland leisten zu können - der Traum jedes Forschers, und er widmete sein Leben ganz der Erforschung der Radioaktivität. Er war als Direktor des 1910 gegründeten Instituts für Radiumforschung der Akademie der Wissenschaften neben dem Neuseeländer Ernest Rutherford (1871-1937) und dem Ehepaar Marie Curie-Sklodowska (18671934) und Pierre Curie (1859-1906) der große Organisator und ausgleichende Vermittler dieser forschenden Neugierde, für die er Schüler und Mitarbeiter begeisterte. In Meyer verbindet sich das breite Erbe seiner Familie zu einer reichen Palette von Begabungen und Fähigkeiten, die ihn als Naturforscher, Organisator, Kommunikator und Humanisten auszeichnen.

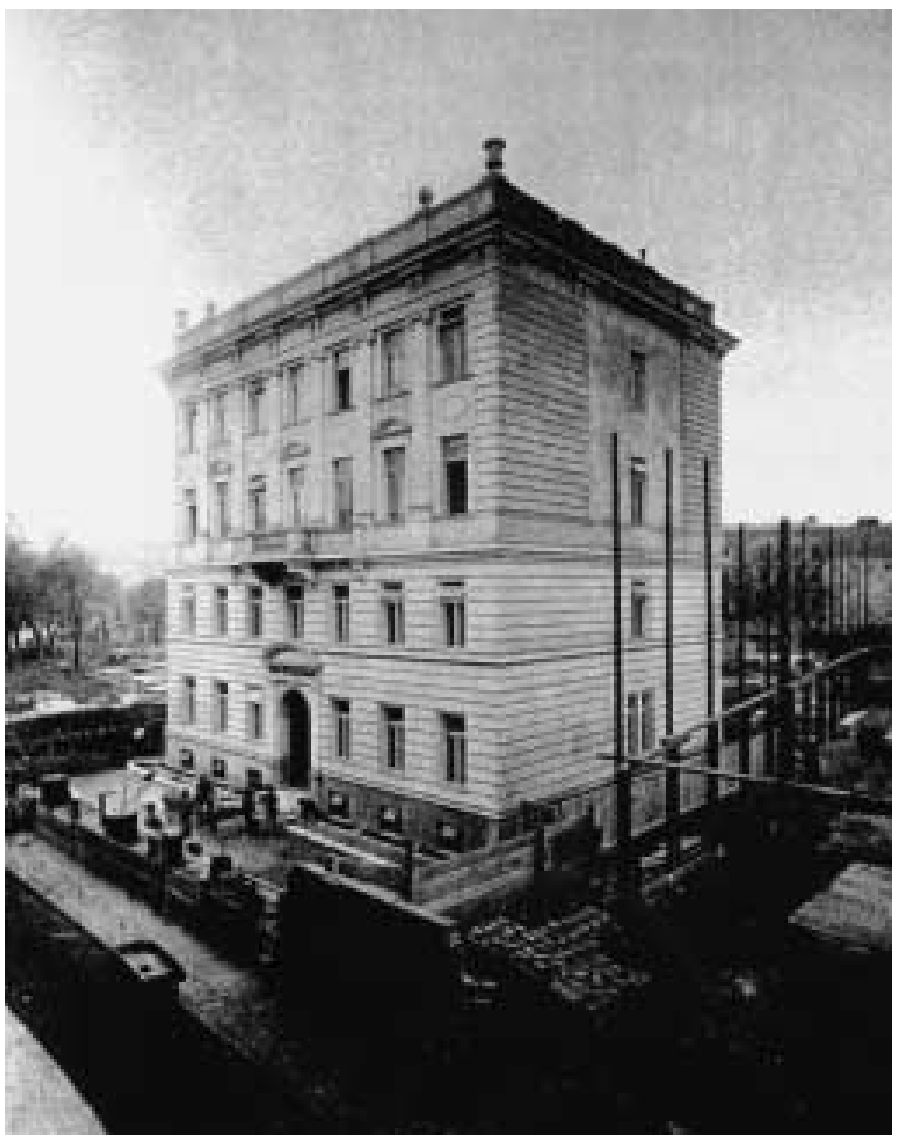

Abb. 3: Das Institut für Radiumforschung im Jahr der Eröffnung 1910. Archiv der Österreichischen Akademie der Wissenschaften.

4 Stefan Meyer und Alexander Wunderer, Grundlagen der Instrumentenkunde für Musikanten und Dilettanten (Wien: Universal-Edition, 1950). 


\section{MEYER - DER PHYSIKER}

Zusammen mit Gustav Jäger (1865-1938) begann Meyer 1897 mit systematischen Messungen der Magnetisierungszahlen von Flüssigkeiten am Institut für theoretische Physik der Universität Wien unter Zuhilfenahme eines von Ludwig Boltzmann (1844-1906) angeschafften Elektromagneten. Die beiden jungen Wissenschaftler lösten einander in diesem Jahr als Assistenten Boltzmanns ab, was dazu berechtigt, Meyer in gleicher Weise sowohl als Schüler Exners als auch Boltzmanns anzusprechen.

In Wolfenbüttel bei Braunschweig beschäftigten sich die beiden deutschen Physiker Julius Elster (1854 - 1920) und Hans Geitel (1855 - 1923) intensiv mit dem 1896 von Antoine Henri Becquerel (1852 - 1908) entdeckten Phänomen von unsichtbaren Strahlen, die nach ihrem Entdecker auch „Becquerel-Strahlung" genannt wurden und wofür in einer Veröffentlichung des Jahren 1898 das Ehepaar Curie den Begriff Radioaktivität prägte. ${ }^{5}$

Anlässlich der „Versammlung der Deutschen Naturforscher und Ärzte“ in München im September 1899, demonstrierte der Braunschweiger Chemiker Friedrich Otto Giesel (1852-1927) von ihm hergestellte Radiumpräparate und deren ionisierende Wirkung. ${ }^{6}$ Meyers Kontaktnahme mit Giesel in München bestimmte fortan sein Lebenswerk. Giesel überließ leihweise dem 27jährigen Wiener Forscher ein für die damaligen Möglichkeiten vergleichsweise „starkes" Radiumpräparat zur Bestimmung der Magnetisierungszahl, also der magnetischen Eigenschaften dieses Elements. Meyer entdeckte in weiteren Versuchen, bei welchen er mit Egon von Schweidler (1873-1948) zusammenarbeitete, an dem von Giesel zur Verfügung gestellten Präparat und an weiteren Präparaten, die Pierre Curie den Wiener Forschern im Juni 1899 überlassen hatte, die magnetische Ablenkbarkeit der „Becquerel-Strahlen“.

Diese für die Natur der radioaktiven Strahlung fundamentale Entdekkung der beiden Wiener Forscher gelang gleichzeitig auch Giesel, sowie Elster und Geitel. Wenig später wurden diese Ergebnisse auch von Henri Becquerel bestätigt. ${ }^{7}$ Die Pariser Radium- und Poloniumpräparate waren ein Geschenk der Curies als Dank für die Hilfe, die Österreich bei der großzügigen Bereitstellung einer bedeutenden Mengen von Uran-Pechblendenrückstän-

5 „Le radio-activité du radium doit donc etre énorme.“ $\mathrm{P}$. und S. Curie und G. Bémont, Comptes Rendus 127 (1898), 1215-1217. In einer Fußnote bedanken sich die Pariser Forscher bei Eduard Suess für die Vermittlung der kostenlosen Überlassung von 100 Kilogramm Pechblendenrückständen aus der Verarbeitung von Uran in St. Joachimstal.

6 St. Meyer, „Zur Geschichte der Entdeckung der Becquerelstrahlen,“ Naturwissenschaften 36 (1949), 129-132. F. Giesel, „Über Radium und Polonium“, Physikalische Zeitschrift I (1899),16-17.

7 St. Meyer und E. v. Schweidler, „Über das Verhalten von Radium und Polonium im magnetischen Felde," Anzeiger der kaiserl. Akad. d. Wiss., mathem.-naturw. Kl., XXII, 3. Nov. 1899, 1-4. St. Meyer und E. v. Schweidler, „Über das Verhalten von Radium und Polonium im magnetischen Felde. (I. Mitteilung), “ Phys. Z., I (1899), 90-91. St. Meyer und E. v. Schweidler, „Weitere Notizen über das Verhalten von Radium im magnetischen Felde," Anzeiger der kaiserl. Akad. d. Wiss, mathem.-naturw. Kl., XXIII, 9. Nov., 1899, 1-2. St. Meyer und E. v. Schweidler, „Über das Verhalten von Radium und Polonium im magnetischen Felde. (II. Mitteilung), "Phys. Z., I (1899), 113-114. F. O. Giesel, „Über die Ablenkbarkeit der Becquerelstrahlen im magnetischen Felde“, Annalen der Physik, 69 (1899), 834-836. J. Elster und H. Geitel, „Über den Einfluß eines magnetischen Feldes auf die durch die Becquerelstrahlen bewirkte Leitfähigkeit der Luft", Verhandlungen der Deutschen Physikalischen Gesellschaft 1 (1899), 136-138 (Sitzung von 5. Mai 1899). H. Becquerel, „Déviation du rayonnement du radium dans un champ électrique“, Comptes Rendus, 130 (1900), 809. Cf. dazu E. Rutherford, Radioactive Transformation (New Haven: Yale University Press, 1906), p. 9. 
den aus dem Bergbau in St. Joachimstal (1898/99 1,1 Tonnen, bis 1906 insgesamt 23,6 Tonnen) geleistet hatte und wodurch überhaupt erst die Voraussetzung geschaffen wurde, die die erstmalige chemische Darstellung von Radium, d. h. die Entdeckung dieses neuen Elements im Dezember 1898 ermöglichte. $^{8}$

In ihren Arbeiten des Jahres 1899 wurde von Meyer und Schweidler der Unterschied der Strahlen des Radiums ( $\beta$-Strahlen) und des Poloniums $(\alpha-$ Strahlen) anhand der unterschiedlichen Ablenkung dieser beiden Strahlenarten im Magnetfeld erstmals richtig gedeutet und damit der entscheidende Nachweis erbracht, dass der Ablenkungssinn identisch mit jenen der Kathodenstrahlen ist, was - in Meyers eigenen Worten - „zur Erkenntnis der korpuskularen Natur dieser Stahlen und Charakteristik der verschiedenen Strahlengattungen führte". ${ }^{9}$ Meyer und Schweidler stellten in ihren Experimenten fest, dass sich die „Poloniumstrahlen“ nicht ablenken ließen; die Feldstärke des von ihnen verwendeten Magneten war dazu nicht stark genug. Mit diesen Arbeiten habilitierte sich Meyer 1900 als Privatdozent an der Universität Wien.

Der von Meyer im Zuge seines Habilitationsverfahrens dem Dekanat der philosophischen Fakultät der Universität Wien vorgelegte Lebenslauf ${ }^{10}$ vom Oktober 1899 hat folgenden Wortlaut:

„Stefan Julius Meyer, am 27. April 1872 in Wien als zweiter Sohn des Herrn Dr. Gotthelf Karl Meyer geboren, evangelisch A. B., erhielt den ersten Unterricht zu Hause, besuchte dann die Volksschule Wien I Doblhoffgasse, sodann das Realgymnasium Wien IX Wasagasse und von der zweiten bis achten Klasse das Landesgymnasium in Horn, NiedOest., woselbst er 1891 das Maturitätszeugnis mit Auszeichnung erhielt. Vom Oktober 1891 bis 1892 Einjährig-Freiwilliger beim $14^{\text {ten }}$ Corps-Artillerie Regiment wurde er 1892 in gleichem Regimente Lieutenant i d. Res. Seine weiteren Studien absolvierte er von 1892 bis 1894 an der philosophischen Fakultät der Universität Wien unter gleichzeitigem Besuche der Collegien der Maschinenbauschule der dortigen technischen Hochschule, verbrachte das Wintersemester 1894/95 an der Universität in Leipzig und von dort zurückgekehrt, die folgende Zeit wieder an der philosophischen Fakultät der Wiener Universität. Am 16. Juli 1896 nach mit Auszeichnung bestandener Prüfung zum Doctor promoviert, arbeitete er im Folgenden im physikalischen Institut der Universität unter Herrn Hofrath Prof. Dr. L. Boltzmann und wurde im Oktober 1897 daselbst Assistent, in welcher Stellung er sich noch befindet."

Das von Meyer aus diesem Anlaß vorgelegte Vorlesungsprogramm ist deshalb beachtenswert, da es zugleich auch mit Bezug auf Meyers eigene Forschungsinteressen zu jener Zeit einen Einblick in die Lehrtätigkeit eines Boltzmannund Exner-Schülers gewährt.

„1.) Die periodischen Eigenschaften der Elemente; 3stündig

Es sollen die wichtigsten physikalischen Eigenschaften der Elemente dargelegt, sowie die Bestimmungsmethoden derselben und deren Theorie auseinandergesetzt werden.

2.) Elektrochemie; 2stündig

Behandlung der Capitel über electrolytische Leitung

Umwandlung chemischer in elektrische Energie und die osmotische Theorie, dann über die Theorie der galvanischen Stromerzeugung und die Polarisation.

8 Wolfgang L. Reiter, „Die Uranlagerstätte in St. Joachimstal und die Radioaktivitätsforschung in Österreich. "Res Montanarum. Zeitschrift des Montanhistorischen Vereins für Österreich. Bd. 8, Leoben1994, $14-23$.

9 St. Meyer, „Die Vorgeschichte der Gründung und das erste Jahrzehnt des Instituts für Radiumforschung, “ in: „Festschrift des Instituts für Radiumforschung anläßlich seines 40jährigen Bestandes (1910 - 1950)“, Sitzungsberichte der mathem.-naturwiss. Kl. Abt. IIa, 159. Bd., 1. - 2. Heft, Wien 1950, p. 2.

10 Österreichisches Staatsarchiv, Akte Stefan Meyer. 
3.) Mechanische Wärmetheorie; 3stündig

Nach Besprechung der allgemeinen Grundlagen, sollen die wichtigsten auf Grund der Anschauungen der kinetischen Gastheorie gewonnenen Sätze entwickelt und erörtert werden.

4.) Thermometrie; 1stündig

Historische Entwicklung der Themperaturbestimmungsmethoden und Besprechung der absoluten Temperaturscala.

5.) Magnetismus; 2stündig

Die hauptsächlichen, experimentell gegebenen Thatsachen sollen besprochen und die verschiedenen Methoden der Feldstärkebestimmung näher behandelt werden.“

Sowohl Elster und Geitel als auch Schweidler (als Schüler Exners) hatten große Erfahrung auf dem Gebiet der atmosphärischen Elektrizität und der Leitfähigkeit in Gasen, die für sie die Untersuchung des ionisierenden Verhaltens der neuen Strahlen unmittelbar interessant machen mußte. Die notwendige Grundvoraussetzung der Wiener Physiker für diese Experimente, nämlich über entsprechendes radioaktives Material zu verfügen, war durch die Kontakte Exners mit Pierre Curie gegeben. Die an Exner's Institut zur Verfügung stehenden radioaktiven Präparate boten daher im Anschluß an Meyers erste Versuche, die magnetischen Eigenschaften des Radiums zu studieren, Meyer und Schweidler die damals nur einer Hand voll Wissenschaftlern offen stehende Möglichkeit, das Ablenkungs-Experiment - ein experimentum crucis - durchzuführen. ${ }^{11}$ Die Existenz des starken Elektromagneten am Boltzmann'schen Institut war zudem die wesentliche apparative Voraussetzung für den Erfolg ihrer Ablenkungsversuche.

Meyer war seit der allerersten Zeit der Kontakte Pierre Curies mit den offiziellen österreichischen Stellen, vor allem mit dem Präsidenten der Akademie, Eduard Suess (1831-1914), zu dessen Familie Meyer freundschaftliche Verbindungen pflegte, sowie mit Exner ganz eng in das laufende Geschehen eingebunden. Meyer schreibt dazu: „So ergab es sich, daß ich von Anbeginn an von allen dreien (Boltzmann, Exner, Suess; W. L. R.) zu Informationen herangezogen wurde, die dieses Fachgebiet betrafen, besonders auch für den Verkehr mit Paris und England." 12 Suess und Exner hatten ihrerseits wiederum enge Verbindungen zu dem Chemiker und Industriellen Carl Joh. Auer v. Welsbach (1858-1929).

Meyers wissenschaftliches Interesse ging von den eigenen frühen Arbeiten zur Bestimmung der Magnetisierungszahlen des Radiums und Poloniums aus und führte ihn zur Untersuchung der Ablenkbarkeit der Polonium- und Radium-Strahlen, die zum Ausgangspunkt seiner lebenslangen Beschäftigung mit der Radioaktivität wurde. Meyer kommt auch später noch auf sein erstes Forschungsinteresse zurück, die Bestimmung von Magnetisierungszahlen, als er 1924 weitere Messungen an seltenen Erdelementen vornimmt, die Arnold Sommerfeld (1868-1951) unter anderem als Grundlage für quantentheoretische Berechnungen zum Atombau der seltenen Erden dienen. ${ }^{13}$

11 Marjorie Malley, „The Discovery of the Beta Particle“, American Journal of Physics 39 (1971),1454-1461.

12 Ref. 9., p. 4.

13 St. Meyer, „Magnetisierungszahlen seltener Erden“, Sitz.ber. d. mathem.-naturwiss. Kl. Abt. IIa, 134, 491-496 und Phys. Zts., 26 (1925), 51-54 und 478-479.

A. Sommerfeld, ,Zur Theorie des periodischen Systems“, Phys. Zts. 26 (1925), 70. Cf. dazu W. Pauli, „Über den Zusammenhang des Abschlusses der Elektronengruppen im Atom mit der Komplexstruktur der Spektren“, Z. f. Phys., 31 (1925), 765. G. v. Hevesy, Die seltenen Erden vom Standpunkte des Atombaues (Berlin: Springer, 1927), p. 41. 
Dies sei deshalb betont, da die Konstellation von Forschungsinteressen der Exner-Schule ${ }^{14}$ auf dem Gebiet der Luftelektrizität und Ionisierung und die physikalischen (Meyer, Schweidler und Jäger) und chemischen (C. Auer von Welsbach und seinen Mitarbeitern Ludwig Haitinger und Carl Ulrich) Untersuchungen der Eigenschaften der seltenen Erdelemente ideale Voraussetzungen für den Einstieg der Wiener Forscher in die Radioaktivitätsforschung schuf. Meyers Persönlichkeit tritt schon hier konturiert hervor, als jener, der fachliche, organisatorische und menschliche Brücken baut und kreativ die Herausforderung neuer Fragestellungen aufnimmt.

Die aktive und maßgebliche Rolle der Kaiserlichen Akademie der Wissenschaften in dieser frühen Phase der Radioaktivitätsforschung mündet 1901 in die Gründung der „Kommission für die Untersuchung der radioaktiven Substanzen“, die unter Exners Vorsitz steht und der unter anderem Suess, Boltzmann, Viktor von Lang (1838-1921) und Auer von Welsbach angehören. ${ }^{15}$ Auf deren Anregung hin werden in den Jahren 1904 bis 1907 in der Gasglühlichtfabrik Auer von Welsbachs in Atzgersdorf bei Wien insgesamt 4 Gramm Radiumchlorid aus Verarbeitungsrückständen des St. Joachimstaler Uran-Bergwerks gewonnen und damit, wie Meyer bemerkt, „das Fundament für systematische wissenschaftliche Tätigkeit auf diesem Gebiet gelegt “ ${ }^{16}$ Dieser für die damaligen Verhältnisse in seiner Menge einmalige „Radiumschatz“ wurde als Morgengabe von der Akademie der Wissenschaften in das 1910 eröffnete Institut für Radiumforschung eingebracht.

Noch sind einige biographische Daten nachzuführen, ehe wir uns Meyers Rolle als Leiter des Instituts für Radiumforschung zuwenden können. 1906/ 07 wird Meyer nach dem tragischen Selbstmord Boltzmanns und bis zur Ernennung von Fritz Hasenöhrl (1874-1915) als dessen Nachfolger die provisorische Leitung des Instituts für theoretische Physik übertragen. 1907 erfolgt seine Zuteilung an das II. Physikalische Institut als Exners Assistent. Im folgenden Jahr wird Meyer zum a. o. Professor ernannt. 1909 wendet er sich Untersuchungen zur Verfärbung und von Lumineszenzerscheinungen von Festkörpern (insbesondere Kunzit und Alkalihalogenide) zu, die durch radioaktive Stoffe hervorgerufen werden, und gibt mit diesen Arbeiten den Anstoß für die ab 1912 am Radiuminstitut schulbildenden Forschungsarbeiten eines Freundes Karl Przibram (1878-1973). ${ }^{17}$ Mit diesen frühen Pionierarbeiten und ihrer Weiterführung durch Przibram werden die ersten Grundlagen für jenes Gebiet geschaffen, das heute als nukleare Festkörperphysik bekannt ist.

Nur streifen kann ich hier die Tatsache, daß Lise Meitner (1878-1968), die bei Exner und Boltzmann studierte und 1905 bei Exner ihre Dissertation abschloß, von Stefan Meyer zu ihren ersten experimentellen Arbeiten auf dem radioaktiven Gebiete angeregt wurde - es entstehen 1906 und 1907 zwei erste Publikationen -, ehe sie im September 1907 nach Berlin ging. ${ }^{18}$

14 Berta Karlik und Erich Schmid, „Franz Serafin Exner und sein Kreis. Ein Beitrag zur Geschichte der Physik in Österreich" (Ref. 2).

15 Richard Meister, Geschichte der Akademie der Wissenschaften in Wien 18471947. Österreichische Akademie der Wissenschaften, Denkschriften der Gesamtakademie, Band I. (Wien: Holzhausens Nfg., 1947).

16 St. Meyer, „Das erste Jahrzehnt des Wiener Instituts für Radiumforschung,“ Jahrbuch der Radioaktivität und Elektronik 17 (1920), 1-29.

17 St. Meyer, „On the change of colour of Kunzite by Becquerel-Radiation, “ Ion I,4 (1909), 260. St. Meyer, „Über das Verhalten von Kunzit unter Einwirkung von Becquerelstrahlung," Phys. Zts., 10 (1909), 483-484.

18 Lise Meitner, „Über die Absorption der $\alpha$ - und $\beta$-Strahlen“, Phys. Z. 7 (1906), 588-590, und dies., „Über die Zerstreuung der $\alpha$-Strahlen“, Phys. Z., 8 (1907), 489-491. 

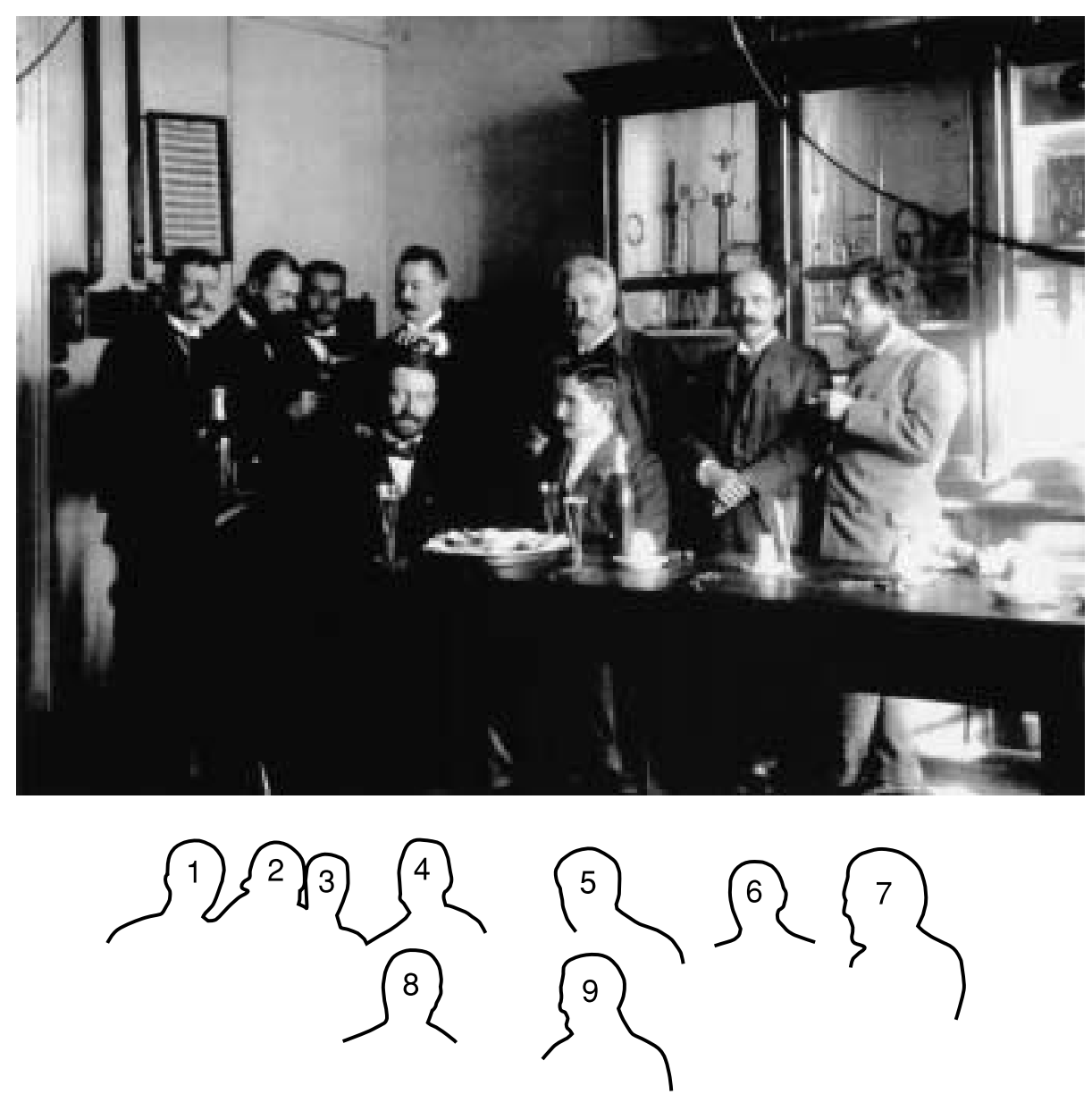

Abb. 4: Feier aus Anlaß der Habilitation von Friedrich Hasenöhrl und Stefan Meyer (1899). 1 Egon von Schweidler, 2 Eduard Haschek, 3 Felix Exner, 4 Gustav

Jäger, 5 Franz S. Exner, 6 Cesar Pomeranz, 7 Anton Lampa, 8 Friedrich Hasenöhrl, 9 Stefan Meyer. Archiv der Österreichischen Zentralbibliothek für Physik.

Wie erfolgreich Meyer und Schweidler bei der Bestimmung und Aufklärung von Zerfallsprodukten des Radiums und der Zuordnung von neu entdeckten radioaktiven Stoffen zu Gliedern schon bekannter Zerfallsreihen waren, dafür zeugt ein Brief Ernest Rutherfords vom 5. November 1904, in dem er seinen Wiener Kollegen zum gelungenen Nachweis der Identität des Radiobleis mit Polonium gratuliert. ${ }^{19}$ Rutherford schreibt: „The results in your last paper on radiolead have been of special interest to me and I congratulate you at your proofs of the identity of the procucts of radiolead with those of the active deposit of radium. There can now be no doubt of their connection. " ${ }^{20}$ In diese Zeit fallen auch die Untersuchungen, welche zeigen, daß Polonium kein stabiles Element ist, sondern mit einer Halbwertszeit von 140 Tagen zerfällt. In der Periode 1904 bis 1907 publiziert Meyer gemeinsam mit Schweidler zehn Arbeiten unter dem Sammeltitel „Untersuchungen über radioaktive Substanzen", die ihn in seiner Rolle eines Pioniers des Fachs etablieren. Alle diese Ar-

19 St. Meyer und E. v. Schweidler, „Untersuchungen über radioaktive Substanzen. V. Mitteilung. Über Radioblei und Radium-Restaktivitäten“, Wiener Sitz. ber. IIa, 114 (1905), 1195-1219.

20 Zitiert nach: A. S. Eve, Rutherford: Being the life and letters of the Rt. Hon. Lord Rutherford, O. M. (Cambridge: Cambridge University Press, 1939), p. 114. St. Meyer, Vorgeschichte (ref. 9), p. 2. 
beiten entstehen noch am alten physikalischen Institut in der Türkenstraße im IX. Wiener Bezirk. ${ }^{21}$

Von der kollegialen Freundschaft zwischen den Pariser und Wiener Forschern wurde schon berichtet. Von gleicher Bedeutung, wenn nicht noch gröBerer, war die fachliche und freundschaftliche Beziehung zwischen Meyer und Ernest Rutherford. Mit der für Meyer charakteristischen diplomatischen Geschicklichkeit gelang es ihm zwischen William Ramsey (1852-1916), damals Rutherfords wissenschaftlichen Konkurrenten in London, und Rutherford zu vermitteln, als beide zur gleichen Zeit an die Wiener Stellen herantraten, man möge ihnen für ihre Experimente radioaktives Material überlassen.

Auf Meyers Intervention hin stellte die Wiener Akademie zu Beginn des Jahres 1908 Rutherford ohne jegliche weitere Bedingungen und völlig zu seiner eigenen freien und uneingeschränkten Verfügung $300 \mathrm{mg}$ Radium als Chlorid zur Verfügung. Diese großzügige Leihgabe ermöglichte Rutherford die ungehinderte Entwicklung seines eigenen Forschungsprogramms. 1911 konnte Rutherford sein Modell des Aufbaus des Atoms, bestehend aus einem positiv geladenen Kern umgeben von negativ geladenen Elektronen, präsentieren, deren Formulierung auf Experimenten beruhte, zu deren Gelingen die von Wien gelieferten radioaktiven Präparate entscheidend beitrugen. $\mathrm{Ru}-$ therford schreibt dazu in einem Brief vom 21. Dezember 1921 an Meyer: „The use of the material has rendred possible the long series of investigations in Radioactivity by myself and my students and has been an invaluable aid in my research." 22

\section{MEyer - DER Organisator, das Institut FÜr Radiumforschung}

Das Jahr 1910 ist wie kein anderes von wichtigen Ereignissen in Meyers Leben geprägt. Anlässlich des 2. Internationalen Kongresses für Radiologie und Elektronik in Brüssel wird die „Internationale Radium-Standard-Kommisson" ins Leben gerufen. Rutherford übernimmt die Päsidentschaft und Meyer wird zum Sekretär der Kommission bestellt. Er übernimmt damit in seinem 38. Lebensjahr eine internationale Funktion, die ihn als Wissenschaftler und Organisator bis zu seinem Lebensende begleiten wird. In dieses Jahr fällt auch seine Heirat mit Emilie Therese Maass, die mütterlicherseits der Prager Familie von Portheim entstammt und ihrerseits mit Meyers Mutter verwandt ist. Die Verleihung des Franz-Joseph-Ordens weist auf eine Leistung hin, die Meyer im öffentlichen Interesse vollbracht hat - die Planungen für das Radiuminstitut.

Im Jahre 1908 hatte der Wiener Rechtsanwalt und Industrielle Karl Kupelwieser der Akademie der Wissenschaften einen Betrag von 500.000 Kronen zur Errichtung und apparativen Ausstattung eines Gebäudes für die physikalische Erforschung des Radiums gewidmet. Seinen Stiftungsbrief beginnt Kupelwieser mit diesen Worten:

„Die Besorgnis, daß meine Heimath „Österreich“ etwa verabsäumen könnte, sich eines der größten ihm von der Natur überlassenen Schätze, nämlich des Minerals Uran-Pech-

${ }^{21}$ K. Przibram, „Erinnerungen an ein altes physikalisches Institut,“ in O. R. Frisch, F. P. Paneth, F. Laves, P. Rosbaud, eds.,Trends in Atomic Physics: Essays dedicated to Lise Meitner, Otto Hahn, Max von Laue on the occasion of their 80th birthday. (New York: Interscience, 1959), p. 2-6.

22 St. Meyer, Vorgeschichte (ref. 9), p. 17. 


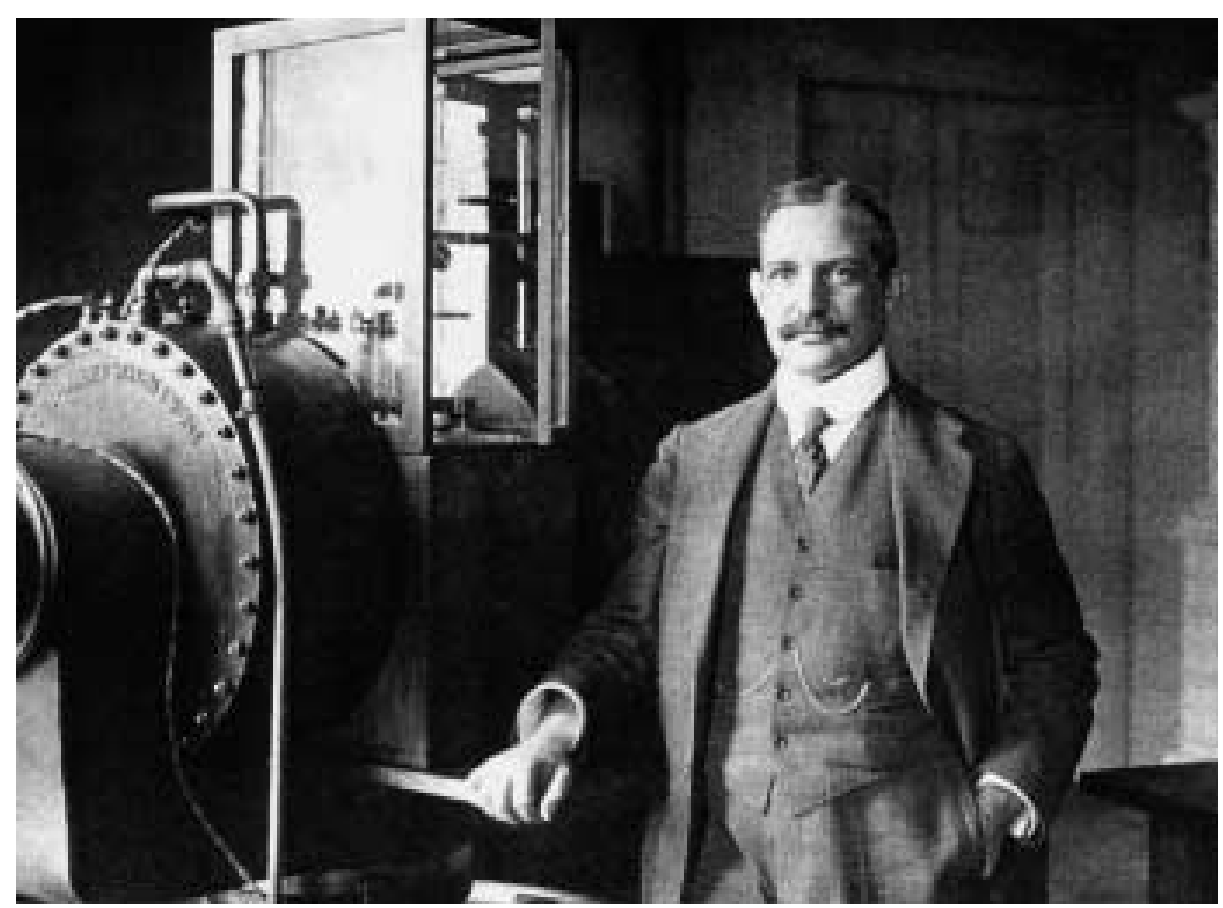

Abb. 5: Stefan Meyer (ca. 1912) im „Magentzimmer“ des Instituts für Radiumforschung. Archiv der Österreichischen Zentralbibliothek für Physik.

blende wissenschaftlich zu bemächtigen, beschäftigt mich schon seit dem Bekanntwerden der räthselhaften Emanationen ihres Produktes „des Radiums“.

Ich wollte, soweit meine Kräfte reichen, zu verhindern trachten, dass mein Vaterland die Schande treffe, daß es eine ihm gewissermaßen als Privilegium von der Natur zugewiesene wissenschaftliche Aufgabe sich habe von Anderen entreißen lassen. Es blieb mir hierzu in unserem etwas schwerfälligen Reiche unter den wirklich schon drängenden Umständen kein anderer Weg, als selbst in die Tasche zu greifen und wenigstens den Pfad zu ebnen versuchen". ${ }^{23}$

In den folgenden beiden Jahren bis zur Eröffnung des Instituts für Radiumforschung am 28. Oktober 1910 war Meyer mit den Planungsarbeiten für die Errichtung und die Ausstattung dieses neuen Instituts beschäftigt. ${ }^{24}$ Welche Herausforderungen Meyer dabei zu bewältigen hatte, erhellt die Tatsache, dass es zu jener Zeit keinerlei Vorbild für den Aufbau, die Einrichtung und die apparative Ausgestaltung dieser innovativen wissenschaftlichen Institution gab. Denn das Radiuminstitut war das erste wissenschaftliche Institut seiner Art weltweit, das sich ausschließlich der Erforschung des Phänomens der Radioaktivität widmen sollte. Meyer entschlug sich dieser Aufgaben in so vorbildlicher Weise, daß das Schwesterinstitut, das Pariser Institut du Radium, das jedoch erst 1915 eröffnet wurde, weitgehend nach dem Wiener Vorbild errichtet und eingerichtet wurde. ${ }^{25}$

23 Archiv der Österreichischen Akademie der Wissenschaften. Akte Karl Kupelwieser. Abschrift Zl. 713 ex 1908. Eine gekürzte Version des Stiftungsbriefes in St. Meyer, „Das erste Jahrzehnt," (ref. 10), 1-29.

${ }^{24}$ Cf. St. Meyer, „Das erste Jahrzehnt,“ (ref. 16), 1-29. Hier findet sich auch eine Planskizze des Instituts.

25 Der Bau des Pariser Instituts wurde 1912 begonnen. Das Institut umfaßte zwei Laboratorien; das eine war der physikalischen und chemischen Untersuchung der radioaktiven Körper, das andere deren biologischer und therapeutischer Verwendung gewid- 
1911 wird Meyer - nicht zuletzt auch in Anerkennung seiner Verdienste beim Aufbau des neuen Institutes - zum wirklichen Extraordinarius ernannt und erhält 1915 den Titel und Charakter eines o. ö. Professors an der Universität Wien. In Anerkennung seiner wissenschaftlichen Leistungen wird ihm 1913 der Lieben-Preis der Akademie der Wissenschaften zuerkannt. Wenn auch Exner von der Akademie zum nominellen Vorstand des Instituts bestellt wurde, so lagen doch alle ganz konkreten Leitungsfunktionen und die Planung der wissenschaftlichen Arbeiten des Instituts bei Meyer.

Die Aufbruchstimmung und die fruchtbare Atmosphäre der ersten Jahre am neuen Institut kann nicht besser beschrieben werden, als dies Georg von Hevesy (1885-1966) in seinen „Erinnerungen an die alten Tage am Wiener Institut für Radiumforschung“ getan hat. ${ }^{26}$ Der Nobelpreisträger für Chemie erzählt von seiner Freundschaft mit dem Wiener Chemiker Friedrich Paneth (1887-1958) und dem Beginn ihrer gemeinsamen Arbeit im Dezember 1912, die zur Entwicklung der „Radio-Indikator-Methode“ führte. „Es waren herrliche, unvergeßliche Zeiten; Hönigschmid arbeitete am Institut, Atomgewichtsbestimmungen von großer Wichtigkeit wurden ausgeführt, Etalons (das sind radioaktive Standard-Präparate für Eichzwecke, W. L. R.) wurden hergestellt, wir erlebten die Entdeckung der Höhenstrahlung“. Auch Hevesys einfühlsame Beschreibung der persönlichen Ausstrahlung Meyers darf hier zitiert werden:

„Stefan Meyer war ja nicht nur der Direktor des Instituts, er war der väterliche Freund aller, die jemals am Institut tätig waren: selbstlos, stets hilfsbereit mit Rat und Tat, von unerschöpflicher Liebenswürdigkeit. Seine Gastfreundschaft kannte keine Grenzen. Die Erinnerung an die zahlreichen angeregten Stunden, die ich in seinem schönen und hochkultivierten Heime verbringen durfte, stehen lebhaft vor mir".

Und fast schwärmerisch erinnert sich Viktor F. Hess (1883-1964) seiner ersten Jahre am Institut: „Als wir im Oktober 1910 einzogen, war ich der erste Assistent. Alles war aufs beste eingerichtet in diesem damals einzigen Institut dieser Art auf der ganzen Welt. Es war eine wahre Freude, über all diese vorzüglichen Apparate und Behelfe verfügen zu können." ${ }^{27}$

Die ersten Jahre des Instituts, für die der Erste Weltkrieg und das Jahr 1918 mit dem Zerfall der Donaumonarchie die einschneidende Zäsur darstellen, waren außerordentlich erfolgreich. Viktor F. Hess, damals Assistent am Institut, gelang 1912 die Entdeckung der kosmischen Strahlung, für die er 1936 mit dem Nobelpreis für Physik ausgezeichnet wurde. Die Entwicklung der Radio-Indikator-Methode durch Hevesy und Paneth ab 1913 am Wiener Institut wurde mit der Verleihung des Nobelpreises für Chemie 1943 an Hevesy gewürdigt. ${ }^{28}$ Darüber hinaus waren es strahlenchemische Arbeiten, methodische Entwicklungen und die Untersuchung und Anwendung der Wirkung radioaktiver Strahlung in der Medizin und in der Festkörperphysik, weiters die von dem Prager Chemiker Otto Hönigschmid am Institut ausgeführten ultragenauen Atomgewichtsbestimmungen und die Herstellung und die von Meyer

met. 1913 wurde auch in Warschau ein Radiuminstitut eröffnet, das unter der Leitung von L. Wertenstein stand; Ehrenvorstand des Instituts war M. Curie.

26 G. von Hevesy, „Erinnerungen an die alten Tage am Wiener Institut für Radiumforschung,“ in: „Festschrift“ (ref. 9), p. 48.

27 Viktor F. Hess, „Persönliche Erinnerungen aus dem ersten Jahrzehnt des Instituts für Radiumforschung, “ in: Festschrift (ref. 9), p. 43.

${ }^{28}$ Hilde Levi, George de Hevesy. Life and Work (Copenhagen: Rhodos, 1985). 
selbst unternommene Eichung von Radium- Standards, die den internationalen Rang des Institutes und seiner Mitarbeiter weiter festigten.

Die organisatorische Tatkraft Meyers, die in dem Wort Freundschaft und Gastfreundschaft sehr traditionell beschrieben wird, kommt auch darin zum Ausdruck, dass das Institut von ihm als eine internationale Begegnungsstätte verstanden wurde. Mehr als $20 \%$ der Mitarbeiter, die in den ersten 25 Jahren am Institut tätig waren, waren ausländische Forscher. Und es gilt eine weitere Tatsache hervorzuheben, die die offene und liberale Haltung Meyers verdeutlicht: der hohe Anteil weiblicher Mitarbeiter am Radiuminstitut, der für die genannte Zeit $30 \%$ beträgt, ein Faktum, das für ein naturwissenschaftliches Fachgebiet auch heute noch Beachtung verdient. Meyer war der unbestrittene, milde und benevolente Vater, der Patriarch der Wiener Radiumfamilie.

Es ist schwierig, der Breite des wissenschaftlichen Lebenswerks Stefan Meyers hier gerecht zu werden. Eine Facette seiner wissenschaftlichen Arbeiten kann nicht unerwähnt bleiben: die vielfältigen Beiträge zur Aufklärung biologischer und medizinischer Wirkungen der Strahlen radioaktiver Stoffe, die Untersuchungen radioaktiver Quellwässer - gemeinsam mit Heinrich Mache (1876-1954) - und deren balneologischen Wirkungen. Kaum ein Gebiet der Radioaktivitätsforschung wurde durch Meyers Arbeiten nicht bereichert. Das wissenschaftliche Oeuvre Meyers umfaßt, neben einer Vielzahl von Arbeiten zu den radioaktiven Zerfallsreihen und zu radioaktiven Altersbestimmungen und zur Kosmogonie, Überlegungen zum Atombau und zum periodischen System und er beschäftigt sich bis in seine späte Schaffensperiode hinein mit Fragen der Zusammenhänge von Atomkern und Elektronenhülle und dem Verständnis des Atomvolumens, i. e. das Verhältnis von Atomgewicht bzw. Ordnungszahl (ein Begriff, dessen Einführung Meyer schon 1906 statt des Atomgewichts vorgeschlagen hatte) zur Dichte der verschiedenen Elemente.

Eine Summe seines wissenschaftlichen CEuvres, dessen produktivste Phase in den Jahren zwischen 1896 und 1916 liegt, bildet das gemeinsam mit Schweidler verfaßte erste umfassende Standardwerk des Gesamtgebiets der Radioaktivitätsforschung in deutscher Sprache, das 1916 unter dem Titel „Radioaktivität" publiziert wird. ${ }^{29}$ Dieses Buch gilt bis heute neben den Publikationen Rutherfords und Marie Curies als Quellenwerk und erschien 1927 in zweiter Auflage.

Die Bedeutung Meyers in seinem Fach läßt sich mit Hilfe zitationsanalytischer Methoden verdeutlichen: Für die Periode 1920 bis 1925 nimmt Meyer unter den meist zitierten Autoren im Fach Kernphysik den Rang 11 ein. Die Ränge 1, 2, 3, 6 und 8 besetzen Bohr, Fajans, Born, Rutherford und Hevesy; die Ränge 12 und 13 Geiger und Ladenburg. Bedenkt man den relativ späten Zeitraum, der dieser Analyse zugrunde liegt, so erkennt man die Bedeutung Meyers für die Radioaktivitätsforschung. ${ }^{30}$

29 St. Meyer und E. v. Schweidler, Radioaktivität (Berlin und Leipzig: B. G. Teubner 1916); 2. erweiterte Auflage 1927.

30 Klaus Fischer, „Die Emigration deutschsprachiger Kernphysiker nach 1933. Eine kollektivbiographische Analyse ihrer Wirkung auf der Basis szientometrischer Daten,“ in: „Exilforschung. Ein internationales Jahrbuch.“ Vol. 6, 1988: Vertreibung der Wissenschaften und andere Themen (München:1988), 44-72. Ders., „Changing Landscapes of Nuclear Physics. A Scientometric Study on the Social and Cognitiv Position of German-Speaking Emigrants within the Nuclear Physics Community 1921-1947,“(Berlin: Springer, 1993). 
Im Jahr der Emeritierung Exners 1920 erfolgte die Ernennung Stefan Meyers zum Ordinarius und gleichzeitig zum selbständigen Vorstand des Instituts für Radiumforschung, eine Funktion die er de facto seit 1910 ausübte. Im folgenden Jahr wird Meyer zum korrespondierenden Mitglied der Akademie der Wissenschaften ernannt, der 1932 seine Ernennung zum wirklichen Mitglied folgte.

Mit Beginn der zwanziger Jahre zieht sich Meyer mehr und mehr von eigenen experimentellen Arbeiten zurück, wenn er auch 1924 noch die bereits erwähnten Messungen der Magnetisierungszahlen der seltenen Erdelemente unternimmt. Die Rolle des Organisators, des Mentors und wohlwollenden Leiters, des toleranten und milden Kritikers seiner Mitarbeiter bestimmen ab dieser Zeit seine Tätigkeit und prägen die Atmosphäre des Instituts. Aus der Sicht einer Mitarbeiterin schreibt dazu Elizabeth Rona (1890-1982):

„The atmosphere at the institute was most pleasant. We were all members of one familiy. Each took an interest in the research of the others, offering help in the experiments and ready to exchange ideas. Friedships developed that have lasted to the present day. The personalitiy of Meyer and that of the associate director, Karl Przibram, had much to do with creating that pleasant atmosphere. Stefan Meyer, one of the pioneers in radioactivity, already had a distinguished career. At the time when I joined the staff of the Radium Institute he scarcely did any research himself, but he did closely follow the research activities of his staff and students, sometimes with more benevolence than criticism. “31

Und Otto Hahn (1879-1968) findet in seinem Nachruf auf Meyer folgende Worte:

„Als einen besonderen Zug wahrhaft kollegialer Treue seinen vielen Mitarbeitern gegenüber habe ich es immer empfunden, daß er jedem Einzelnen weitgehende Freiheit in seiner Arbeit gewährte, ihn immer allein publizieren ließ, obgleich er ja doch bei sehr vielen Arbeiten der geistige Anreger war." 32

Eine Reihe von jungen Mitarbeitern beginnen am Radiuminstitut mit neuen Entwicklungen. Um den schwedischen Ozeanographen Hans Pettersson bildet sich ab 1922 eine kleinen Gruppe, die Untersuchungen zur Kernzertrümmerung, heute als künstliche Kernumwandlung bezeichnet, nachgehen. Die Ambitionen dieser Gruppe um Pettersson und Gerhard Kirsch (1890- 1956) führen zu einer Kontroverse mit dem Cavendish Laboratory in Cambridge, da die Ergebnisse der Wiener Gruppe mit einer am gleichen Problem arbeitenden Gruppe um Rutherford mit James Chadwick (1891-1974) und Patrick M. S. Blackett (1897-1974) in Widerspruch stehen. Die experimentellen Resultate der Wiener Gruppe erweisen sich schließlich aufgrund wesentlicher methodischer Mängel als nicht haltbar. Die Kontroverse zwischen Wien und Cambridge des Jahre 1927 wird mit viel diplomatischem Geschick besonders auf Meyers Seite in Abstimmung mit Rutherford applaniert. Doch der gute Ruf des Radiuminstituts war durch die allzu forsche Haltung der Wiener bei der Interpretation ihrer experimentellen Ergebnisse und vielleicht auch aufgrund allzu großer Nachsicht Meyers für seine Mitarbeiter empfindlich getrübt. ${ }^{33}$ Otto Robert Frisch (1904-1979), Lise Meitners Neffe, berichtet dazu:

31 Elizabeth Rona, „How It Came All About. Radioactivity, Nuclear Physics, Atomic Energy“, ORAU 137, 1978, pp. 15-16.

32 Otto Hahn, „Stefan Meyer“, Zeitschrift für Naturforschung 5a (1950) 407.

${ }^{33}$ Roger H. Stuewer, „Artificial Disintegration and the Cambridge-Vienna Controversy“, in Peter Achinstein and Owen Hannaway, eds., Observation, Experiment, 
„Vienna is where I began my career and it was in those days a sort of enfant terrible of nuclear physics. Several physicist were claiming that not only nitrogen and one or two others of the light nuclei could be disintegrated by alpha particles but that practically all of them could and did give many more protons than anybody else could observe. I still do not know how they found these wrong results. Apparently they employed students to do the counting without telling them what to expect. On the face of it, that operation appears to be a very objectiv methode because the student would have no bias; yet the students quickly developed a bias towards high numbers because they felt that they would be given approval if they found lots of particles. Quite likely this situation caused the wrong results along with a generally uncritical attitude and considerable enthusiasm over beating the English at their own game.

I still remember when I left Vienna at just about that time (after having escaped the duty of counting scintillations). My supervisor, Karl Przibram, told me with sadness in his voice, „You will tell the people in Berlin, won't you, that we are not quite as bad as they think?" I failed to persuade them. "34

Von den weiteren Arbeitsgebieten, die ab Mitte der zwanziger Jahre und in den dreißiger Jahren am Institut betrieben wurden, seien besonders zwei erwähnt, da sie über das engere Fachgebiet der Radioaktivitätsforschung hinaus von allgemeiner Bedeutung für weitere experimentelle Entwicklungen in der Physik wurden. Es sind die schon erwähnten Arbeiten von Karl Przibram und seiner Schüler (hier ist vor allen Franz Urbach (1902-1969) zu nennen) auf dem Gebiet der Beeinflussung von Festkörpern durch radioaktive Strahlung. Przibram erarbeitet die ersten Grundlagen zu den Verfärbungsmechanismen von Kristallen und die Abhängigkeit dieser von den Eigenschaften der Kristallstruktur und ihm gelingen die Entdeckung der Radiophotolumineszenz (1921) und grundlegende Erkenntnisse zur Radiothermolumineszenz. Die Summe dieses Forscherlebens zog Przibram in seinem Buch „Verfärbung und Lumineszenz", das 1953 erschien und zu den Standardwerken der Festkörperphysik zählt. ${ }^{35}$ Zum anderen sind hier die bahnbrechenden Arbeiten zur Entwicklung der photographischen Nachweismethode in der Kernphysik zu nennen.

\section{NeUe PhysiK}

Ab Mitte der 20er Jahre beschäftigt sich Marietta Blau (1894-1970) am Institut, mit methodischen Verbesserungen des photographischen Nachweises von Strahlung. In grundlegenden Arbeiten wird diese Methode von ihr zu einem quantitativen Instrument der Messung von Kernreaktionen entwickelt. Bevor sie im Jahre 1938 Wien verläßt, gelingt ihr - gemeinsam mit ihrer Mitarbeiterin Herta Wambacher (1903-1951) - mit dieser Methode erstmals der Nachweis von sogenannten Zertrümmerungssternen, die durch das Zerplatzen von Atomkernen in der photographischen Schicht nach Beschuß durch hochenergetische Teilchen der Höhenstrahlung hervorgerufen werden. Mit der von Blau zur methodischen Reife gebrachten photographischen Nachweismethode wurde der Kernphysik ein neues, einfachen und billiges Nachweisverfahren an die Hand gegeben, das mit der Entdeckung neuartiger Elementarteilchen und hochenergetischer Prozesse die ersten Schritte hin zur

and Hypothesis in Modern Physical Science (Cambridge, Mass.: MIT Press 1985), 239-307.

34 Otto R. Frisch, „The Discovery of Fission: How It All Began“, Physics Today 20 (November 1967), p. 43.

35 Karl Przibram, Verfärbung und Lumineszenz (Wien: Springer-Verlag, 1953). 
Hochenergiephysik einleitete. Die Exposition photographischer Platten in Victor F. Hess' Labor zur Untersuchung der Höhenstrahlung am Hafelekar bei Innsbruck und Blaus Entdeckung erster hochenergetische Kernprozesse stießen das Tor zu neuer Physik auf.

Mit Beginn der dreißiger Jahre endet die Pionierzeit der Radioaktivitätsforschung und es brach eine neue Zeit an, die Ära der Kernphysik. Nachdem zur Mitte der zwanziger Jahre die moderne Quantentheorie in ihrer bis heute gültigen Form durch Erwin Schrödinger, Werner Heisenberg, Max Born und Paul A. M. Dirac formuliert worden war, die zunächst eine Beschreibung der Phänomene der Atomphysik war, sich aber auch für den Bereich des Atomkerns bewährte, erfolgten ab 1932 die ersten beeindruckenden Bestätigungen dieser bis heute scheinbar paradoxen Naturbeschreibung. 1932 entdeckte Carl D. Anderson in der kosmischen Höhenstrahlung ein positiv geladenes Elektron und damit die erste Form von Antimaterie, wie sie im Jahr zuvor von Dirac theoretisch postuliert worden war. Im gleichen Jahr entdeckte Chadwick das Neutron, ein elektrisch neutrales Teilchen, und damit den entscheidenden neuen Baustein - neben dem schon bekannten elektrisch geladenen Proton für das Verständnis des Aufbaus des Atomkerns, wie Heisenberg noch im gleichen Jahr richtig erkannte. Im Jahr darauf formulierte Enrico Fermi seine Theorie des Betazerfalls und schlug darin die Existenz einer schwachen Kernkraft vor, die die Existenz des von Wolfgang Pauli 1930 postulierten spuckhaften Teilchens, des Neutrinos, inkludierte. Schließlich gelang Irène und Frédéric Joliot-Curie 1934 der Nachweis des ersten künstlichen radioaktiven Elements, einer radioaktiven Form des Elements Phosphor. Damit war der Traum der Alchimisten wahr geworden, Elemente verwandeln zu können.

Zur 37. Hauptversammlung der Deutschen Bunsengesellschaft für angewandte physikalische Chemie, die vom 16. bis 19. Mai 1932 in Münster statt-

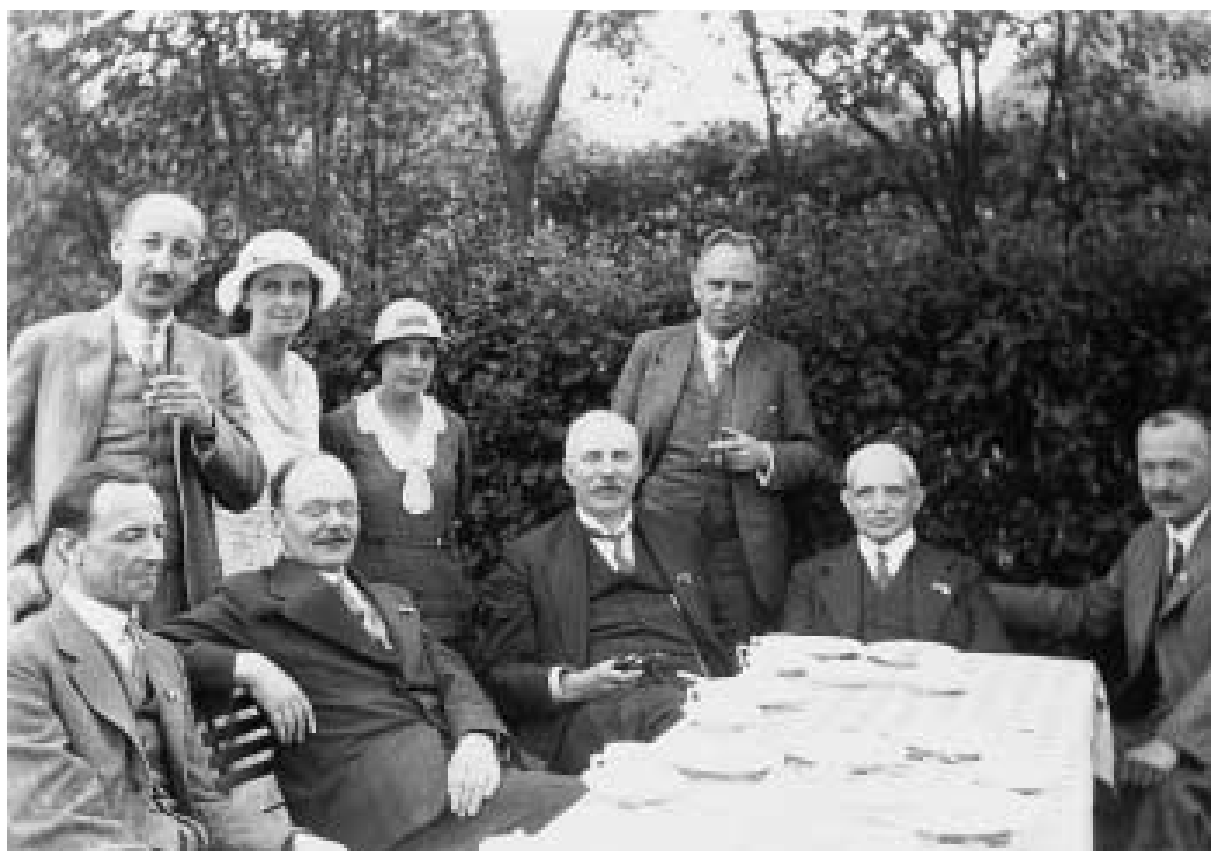

Abb. 6: 37. Hauptversammlung der Deutschen Bunsengesellschaft für angewandte physikalische Chemie, Münster 1932. Von links nach rechts: stehend Georg von Hevesy, Pia von Hevesy (?), Lise Meitner, Otto Hahn; sitzend Frédéric Joliot, Heinrich Mache, Ernest Rutherford, Stefan Meyer, Karl Przibram. Archiv der Österreichischen Zentralbibliothek für Physik. 
fand und ganz unter dem Eindruck der Entdeckung des Neutrons durch James Chadwick stand, trafen sich ein letztes Mal auch die Pioniere der Radioaktivitätsforschung bevor ein Jahr später durch die Machtergreifung Hitlers in Deutschland ein zweites Mal in diesem Jahrhundert die persönlichen Bande durchtrennt wurden. Das Hauptthema der Tagung war dem Gebiet der Radioaktivität gewidmet und dies bot Gelegenheit zum Wiedersehen. Gekommen waren Rutherford und seine Schüler Chadwick, Hans Geiger und Otto Hahn; Wien war mit Meyer und Przibram vertreten, sowie den alten Bekannten der frühen Wiener Zeit, Meitner und Hevesy. ${ }^{36}$

Die Herausforderungen der jüngsten Entdeckungen, insbesondere der des Neutrons, und die Produktion künstlicher radioaktiver Stoffe werden am Institut für Radiumforschung sofort experimentell aufgegriffen, parallel zu den Arbeiten der Gruppe um Fermi in Rom, dem Ehepar Joliot-Curie in Paris und Otto Hahn und Lise Meitner in Berlin. Noch im Jahre 1934 besuchen Irène und Frédéric Joliot-Curie Wien, um von ihren bahnbrechenden Arbeiten zu berichten. Für die Entdeckung der künstlichen Radioaktivität erhielt das Forscherpaar den Nobelpreis für Chemie des Jahres 1935. Der Besuch des Ehepaars kann wohl als letzte, über den engen Fachbezug hinausgehende Demonstration der internationalen Verbundenheit der radioaktiven Forschergemeinde mit Wien und besonders mit Stefan Meyer qualifiziert werden, ehe im März 1938 nicht nur die Existenz Österreichs ausgelöscht, sondern auch - mit spürbaren Folgen bis heute - das wissenschaftliche Leben zerstörte wurde.

\section{DAS JAHR 1938}

Am 22. April 1938 erging vom Bundesministerium für Unterricht an das Dekanat der philosophischen Fakultät der Universität Wien der Erlaß, der mit „sofortiger Wirkung“ für 65 Mitglieder des Lehrkörpers dieser Universität ihre akademische Tätigkeit für beendet erklärte ${ }^{37}$ An erster Stelle der „beurlaubten“ Professoren steht der Name Stefan Meyers, „dieser bis zur Entscheidung über sein Ansuchen um Versetzung in den dauernden Ruhestand“. 38

Meyer hatte am 18. März 1938, sieben kurze Tage nach dem Einmarsch der Hitlertruppen in Österreich, ein Gesuch um Versetzung in den dauernden Ruhestand an die Fakultät gerichtet, in dem er ausführte, er habe „trotz aller meiner für immer treuen Verbundenheit mit meinem Vaterland und trotz der langjährigen Stellung an der Universität die Empfindung ..., nicht mehr in meinem Amte als Universitätsprofessor am Platze zu sein, ...". Auch verzichtet Meyer "freiwillig“ auf seine Mitgliedschaft in der Akademie der Wissenschaften, wohl um der Demütigung eines drohenden Ausschlusses zu entge-

36 O. Erbacher und K. Philipp, „Die Radioaktivität auf der Bunsentagung in Münster“, Naturwissenschaften, 32 (1932), pp. 586.

37 Bundesministerium für Unterricht, Gz. 12474/I/1b aus 1938. Univ. Wien, phil. Fak., Personalmaßnahmen. Österreichisches Staatsarchiv, Allg. Verwaltungsarchiv. Die "Verordnung zur Neuordnung des österreichischen Berufsbeamtentums" vom 31. 5. 1938 (RGBL 1938 I, 607) enthielt im $§ 3$ die Bestimmung, nach der ,jüdische Beamte, Beamte, die jüdische Mischlinge sind und Beamte, die mit einer Jüdin (einem Juden) verheiratet sind", in den Ruhestand zu versetzen sind.

38 Bundesministerium für Unterricht, Gz. 9492/II/6 aus 1938. Univ. Wien, phil. Fak., ord. Prof. Stefan Meyer, Versetzung in den dauernden Ruhestand. Österreichisches Staatsarchiv, Allg. Verwaltungsarchiv. 


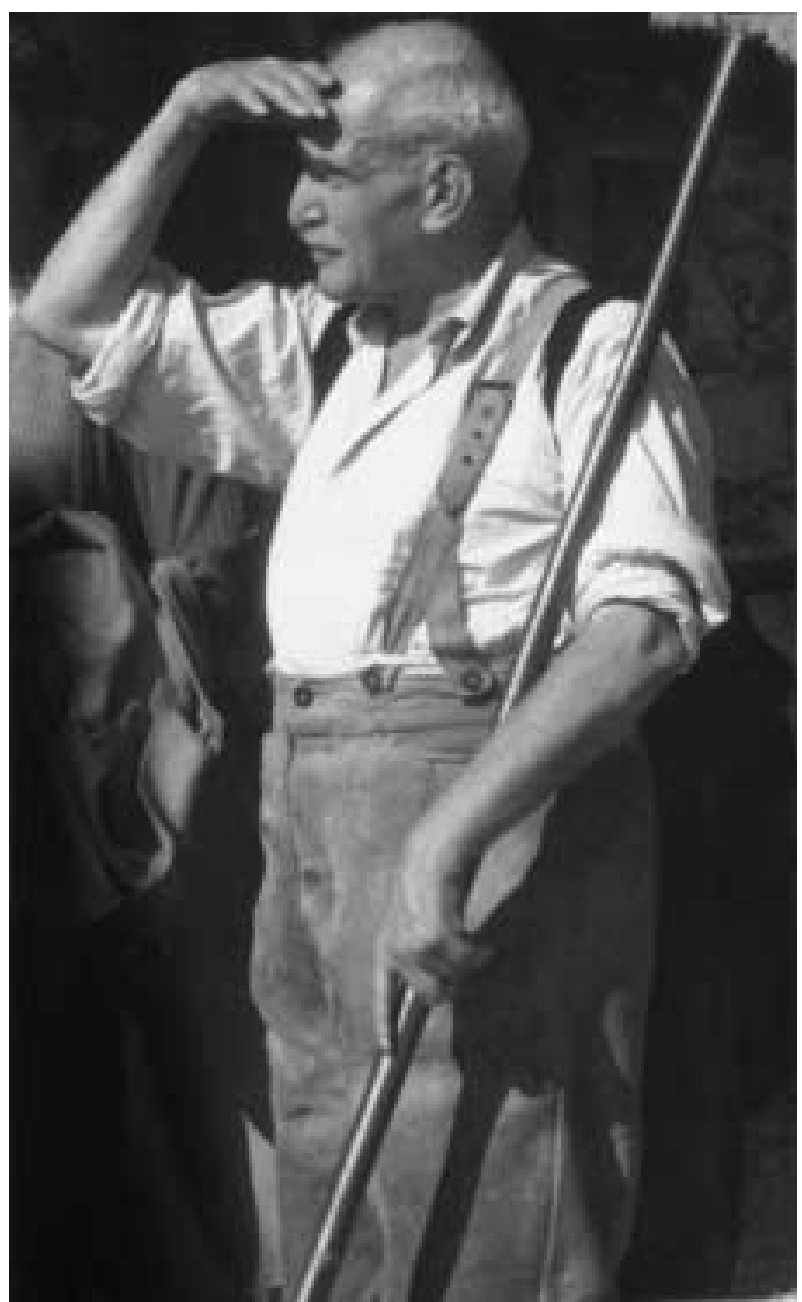

Abb. 7: Stefan Meyer auf der Katrin bei Bad Ischl (ca. 1946). Archiv des Verfassers.

hen. ${ }^{39}$ Vielleicht erhoffte Meyer für sich und seine Familie durch einen weitgehenden Rückzug ins Privatleben sich vor dem Zugriff der Nationalsozialisten bewahren zu können. In welch verzweifelter Situation sich die Angehörigen seiner Familie durch die Machtübernahme der NS-Behörden befanden, erfuhr Meyer am Schicksal seiner jüngsten Schwester Anna (1878-1938), die am 27. März durch Selbstmord aus dem Leben schied. Anna Meyer, die mit der Übersetzung russischer Volksmärchen literarisch hervorgetreten war, bekleidete aufgrund ihrer hervorragenden Kenntnis der russischen Sprache eine wichtige Funktion bei der Fremdenpolizei in Wien.

Meyer und sein Stellvertreter als Institutsdirektor, Przibram, waren noch als „Gäste“ am Institut geduldet, bis auch diese bescheidene Arbeitsmöglichkeit durch eine gelenkte Kampagne aus dem Kreis der früheren Mitarbeiter ihr Ende fand. Meyer zieht sich mit seiner Familie in das Sommerdomizil nach Bad Ischl zurück; Sohn Friedrich wird noch im September 1938 bei Verwandten in England in Sicherheit gebracht. Kollegen aus besseren Tagen, wie Irène und Frédérik Joliot-Curie und Georg von Hevesy, vor allem aber Lise

${ }^{39}$ Meyer wird ebenso wie die korrespondierenden Mitglieder V. F. Hess, E. Schrödinger und V. M. Goldschmidt am 24. November 1938 aus der Preussischen Akademie der Wissenschaften ausgeschlossen. 
Meitner und Hans Pettersson waren bis 1942 intensiv bemüht, die notwendigen Voraussetzungen für eine Emigration der Familie Meyer zu schaffen. ${ }^{40}$ Obwohl Meyer zur Emigration schließlich bereit gewesen zu sein scheint, verwehrte die restriktiver gewordene Ausreisepolitik der Nazibehörden potentiellen Emigranten diesen letzten Ausweg.

Von allen wissenschaftlichen Arbeitsmöglichkeiten abgeschnitten, schrieb Meyer in dieser prekären Situation, die höchst unvollständig mit dem Wort „innere Emigration“ beschreibbar ist, das schon erwähnte Buch über „Instrumentenkunde“ und übersetzte das Buch „Atlantis und Atlantik“ von Hans Pettersson ins Deutsche, wofür er die schwedische Sprache erlernte. ${ }^{41}$

In Folge des Anschlusses Österreichs an Deutschland im März 1938 verlor ungefähr ein Viertel der wissenschaftlichen Mitarbeiter des Instituts ihre Position: Stefan Meyer, Karl Przibram, Marietta Blau, Elisabeth Rona, Anni und Franz Urbach, Eduard Jahoda, Gustav Kürti und Stefan Pelz; ihre akademischen Stellungen, die sie inzwischen an anderen Universitäten innehatten, mußten die ehemals am Institut tätigen Erwin Schrödinger, Viktor F. Hess, Georg von Hevesy, Fritz Paneth, Otto R. Frisch und Victor F. Weisskopf verlassen und in die Emigration gehen. ${ }^{42}$

\section{Meyers LetZTE JAHRE}

Zusammen mit einem in die Gegebenheiten der Familie Meyer eingeweihten Gemeindesekretär in Bad Ischl kommt vor allem Stefan Meyers Tochter Agathe, ihrer Umsicht und ihrem diplomatischen Geschick das Verdienst zu, daß die Familie die Zeit der nationalsozialistischen Herrschaft - bildlich gesprochen auf „Tauchstation“ - überleben konnte. In langen Wanderungen in der von ihm so geliebten Bergwelt des Salzkammerguts und beim Holzhacken für den Bedarf der langen Winter fand die Meyer eigene Zähigkeit ihren Ausdruck, die Zeit des völligen Abgeschnittenseins zu überdauern.

Sofort nach der Befreiung Österreichs 1945 kehrt der nunmehr 73jährige Meyer wieder ans Radiuminstitut zurück in den aktiven Dienst als Honorarprofessor und wird auch seitens der Akademie der Wissenschaften als wirkliches Mitglied rehabilitiert. Im Jahr seiner Versetzung in den dauernden Ruhestand 1947 erscheint der Band „Richtig schauen. Für Maler, Konstrukteure, Fotografen und Bilderfreunde“, das Ergebnis sowohl seiner Interessen für physikalisch-optische Phänomene als auch seiner kunsthistorischen Reflexionen. ${ }^{43}$ Neben dieser Beschäftigung mit der Theorie des Sehens finden wir Meyer noch in seinen letzten Jahren lebhaft mit Problemen der Systematik des Aufbaus der Atomkerne und Planungen zu einer Neuauflage der „Radioaktivität“ beschäftigt. Mit einer Arbeit „Zum Problem des räumlichplastischen Sehens“, die am 13. Oktober 1949 erscheint, schließt dieser Na-

40 Ruth Lewin Sime, Lise Meitner: a life in physics. (Berkeley, Los Angeles, London: University of California Press, 1996), pp. 287-88.

41 Hans Pettersson, Atlantis und Atlantik. Ins Deutsche übertragen von St. Meyer (Wien: Springer-Verlag, 1948).

42 Wolfgang L. Reiter, „Österreichische Wissenschaftsemigration am Beispiel des Instituts für Radiumforschung der Österreichischen Akademie der Wissenschaften, " in Friedrich Stadler ed., Vertriebene Vernunft II, Emigration und Exil Österreichischer Wissenschaft (Wien und München: Jugend und Volk, 1988).

43 St. Meyer, Richtig Schauen. Für Maler, Konstrukteure, Photographen und Bilderfreunde (Wien: Springer-Verlag, 1947). 
turforscher, dem noch der Blick auf das Ganze eigen war, sein wissenschaftliches Oeuvre von insgesamt nahezu 200 Arbeiten.

Kurz vor Vollendung seines 78. Lebensjahrs stirbt Stefan Meyer am 29. Dezember 1949 in Bad Ischl an Herzversagen. Am Neujahrstag des Jahres 1950 wird Meyer in der Ischler Familiengruft beigesetzt.

Aus Anlass seiner Teilnahme bei dem Symposiums zum 100. Geburtstag von Wolfgang Pauli jun. ${ }^{44}$ hat Victor F. Weisskopf, der einst am Radiuminstitut mit Franz Urbach bei Untersuchungen des optischen Verhaltens von gedopten Kristallen zusammengearbeitet hatte ${ }^{45}$, die Worte gesprochen „Wissen ohne Mitgefühl ist unmenschlich“. Ich setze diese lapidare Bemerkung an das Ende dieser Skizze von Person und Werk Stefan Meyers. ${ }^{46}$

\section{Danksagung}

Der Autor ist Frau Dr. Koss-Rosenqvist, Bad Ischl, für die Bereitstellung der Separata zur Erstellung des Verzeichnisses der Schriften Stefan Meyers und Professor Frederic Meyer, Exeter, U.K., für bereitwillige Auskünfte während vieler Jahre zu tiefem Dank verpflichtet. Dem Direktor der Österreichischen Zentralbibliothek für Physik, HR Dr. W. Kerber und seinen Mitarbeiterinnen und Mitarbeitern danke ich für die unermüdliche und prompte Hilfe bei den Literaturrecherchen. Für vielfältige Diskussionen und Anregungen zu Leben und Werk von St. Meyer bin ich Professor Roger H. Stuewer, Minneapolis, zu besonderem Dank verpflichtet.

44 „Wolfgang Pauli und die Physik des 20. Jahrhunderts. Symposium aus Anlaß der Wiederkehr des 100. Geburtstags von Wolfgang Pauli jun.", veranstaltet von der Universität Wien, der Österreichischen Physikalischen Gesellschaft und dem Internationalen Erwin Schrödinger Institut für Mathematische Physik, Wien, 12.-13. April 2000.

${ }^{45}$ Weisskopf, Joy of Insight. Passions of a Physicist (New York: Basic Books, 1991), p. 25.

${ }_{46}$ Auf die nachstehend angeführten Nachrufe sei hier zusammenfassend hingewiesen: Otto Hahn, Stefan Meyer, Z. Naturforschg. 5a (1950) 407. Karl Przibram, in: Die Feierliche Inauguration des Rektors der Wiener Universität für das Studienjahr 1950/51. (Wien: Selbstverlag der Universität, 1950), p. 51-52. Karl Przibram, Stefan Meyer. Almanach der Österreichischen Akademie der Wissenschaften. (Wien, 1951), p. 340 - 352. F. A. Paneth, Prof. Stefan Meyer, Nature 165 (1950), p. 548. Robert W. Lawson, Nature 165 (1950), p. 549. 


\section{ANHANG}

\section{Verzeichnis der Schriften Stefan Meyers}

Im Anhang wird ein vollständiges Verzeichnis der Schriften Stefan Meyers vorgelegt, einschließlich von publizierten Vorträgen, Nachrufen, Artikel in Zeitungen, sowie der von ihm (mit)verfaßten Handbuchartikel, Lehrbuchbearbeitungen, Bücher und Übersetzungen.

Ausgangspunkt für die Erstellung des Verzeichnisses war ein von Meyer selbst verfaßtes Schriftenverzeichnis seiner Arbeiten. Dieses Verzeichnis wurde im einzelnen mit den Originalliteraturstellen verglichen, in vielen Fällen korrigiert sowie in einigen wenigen Fällen durch weitere Arbeiten ergänzt.

Für die Eintragung in das Verzeichnis der Schriften Stefan Meyers wurden die Arbeiten fortlaufend numeriert und nach dem Jahr ihres Erscheinens geordnet. Weitgehend bzw. überwiegend textidentische Arbeiten, die in verschiedenen Journalen oder Reihen veröffentlicht wurden, werden mit der gleichen Zahl unter Hinzufügung einer alphabetischen Zählung angeführt; dieselbe Zählung wurde auch im Falle kurzer Nachträge (Ergänzungen) verwendet.

Bei der Ordnung des Schriftenverzeichnis wurde auf eine weitere Gliederung als die der zeitlichen Zuordnung verzichtet, um das hier erstmals vorgelegte Gesamtverzeichnis möglichst übersichtlich zu halten, die Auffindbarkeit von Einzelarbeiten zu erleichtern sowie den zeitlichen Kontext zu wahren.

Die in das von Meyer erstellte Verzeichnis aufgenommenen Arbeiten der Nummern 144 und 145 liegen nur als Manuskripte vor und konnten darüberhinaus in der Literatur nicht aufgefunden werden. Weiters konnte die Nummer 123 der von Meyer unter dem dort angegebenen Titeln in das Verzeichnis aufgenommenen Arbeit in der Literatur nicht verifiziert werden. Die Redaktion der „Boltzmann-Festschrift“ (1904) sowie die Bearbeitungen von F. Lecher's „Lehrbuchs für Physik“ wurden nicht mit eigener Zählung in das Verzeichnis aufgenommen

Für den Zeitraum 1896 bis 1951 konnten insgesamt 197 Eintragungen in das Schriftenverzeichnis vorgenommen werden. Die beiden 1951 posthum veröffentlichten kunsthistorischen Skizzen waren als Manuskripte schon 1933 fertiggestellt, von Meyer selbst jedoch nicht zum Druck befördert worden.

Stefan Meyer hat eine größere Anzahl von Publikationen gemeinsam mit anderen Autoren veröffentlicht (insgesamt 75, das sind $38 \%$ aller Arbeiten), wobei die Mehrzahl der Arbeiten zusammen mit Mitarbeitern des Physikalischen Instituts der Universität Wien (bis 1910) bzw. des Radiuminstituts (ab 1911) entstand.

Im Folgenden wird dazu eine tabellarische Übersicht der Koautoren, das erste und letzte Jahr der gemeinsamen Publikationstätigkeit, sowie die Anzahl der gemeinsamen Publikationen gegeben.

Unter den Koautoren Meyers ragen drei Namen heraus, mit welchen zusammen $70 \%$ der Arbeiten mit Koautorschaft verfaßt wurden: Egon v. Schweidler (mit 33 gemeinsamen Arbeiten), Heinrich Mache (mit 11 gemeinsamen Arbeiten) und Gustav Jäger (mit 8 gemeinsamen Arbeiten).

Die enge Zusammenarbeit mit G. Jäger in den Jahren 1897-1901 bezog sich auf das Gebiet des Magnetismus, bzw. der Bestimmung der Magnetisierungszahlen verschiedener Stoffe, Arbeiten, die Meyer unmittelbar 1899 über den Versuch der Bestimmung der Magnetisierungszahl des Poloniums und 


\begin{tabular}{lccc}
\hline Name (Geburts-, Todesjahr) & \multicolumn{2}{c}{ erstes Jahr } \\
der Publikation & $\begin{array}{c}\text { letztes Jahr } \\
\text { Anzahl }\end{array}$ & Anstav Jäger (1865-1938) \\
Egon v. Schweidler (1873-1948) & 1897 & 1901 & 8 \\
Heinrich Mache (1879-1954) & 1899 & 1927 & 33 \\
Viktor F. Hess (1883-1946) & 1905 & 1929 & 11 \\
Ludwig Haitinger (1860-1945) & 1911 & 1919 & 4 \\
Karl Peters (?-?) & 1911 & 1911 & 1 \\
Otto Hahn (1879-1968) & 1911 & 1911 & 1 \\
Karl Przibram (1878-1973) & 1912 & 1912 & 1 \\
Fritz Paneth (1887-1958) & 1912 & 1922 & 3 \\
Robert W. Lawson (1890-1960) & 1912 & 1914 & 3 \\
Carl Ulrich (?-?) & 1916 & 1916 & 1 \\
Erhard Suess (1871-1937) & 1923 & 1923 & 1 \\
Alexander Wunderer (?-?) & 1930 & 1930 & 1 \\
Agathe Koss-Rosenqvist(1915-) & 1946 & 1948 & 2 \\
Hans Benndorf (1870-1953) & 1947 & 1947 & 3 \\
Franziska Seidl (1892-1983) & 1948 & 1948 & 1 \\
\hline
\end{tabular}

Radiums (Nr. 14 und 14a) zur Beschäftigung mit dem neuen Gebiet der Radioaktivität führten (Nr. 15 und folgende). Die daran anschließende Periode der so fruchtbaren Zusammenarbeit mit Egon v. Schweidler erbrachte das Meyer'sche Hauptoeuvre und fand ihre Zusammenfassung in dem von den beiden Autoren gemeinsam verfaßten Standardwerk „Radioaktivität" (1. Auflage 1916, 2. erweiterte Auflage 1927). Die 1905 beginnende Zusammenarbeit mit Heinrich Mache war dem Gebiet der Radioaktivität von Quellwässern gewidmet, ein Bereich der Radioaktivitätsforschung, dem Meyer schon früh seine Aufmerksamkeit zuwandte und das in weiterer Folge auch in einer Reihe von Arbeiten zur Balneologie und zur damals reges Interesse beanspruchenden Radiumemanationstherapie Ausdruck fand.

Die Zusammenarbeit mit V. F. Hess (4 gemeinsame Arbeiten), dem ersten Assistenten (Adjunkt) am 1910 eröffneten Wiener Radiuminstitut, betrifft vor allem methodische Fragen der Strahlenwirkungen; sie wird 1920 durch den Ruf Hess' als Extraordinarius an die Universität Graz beendet.

Erwähnenswert für die frühe Periode (bis 1914) ist weiters die Zusammenarbeit mit dem Chemiker Fritz Paneth und dem Physiker Karl Przibram. Während die Zusammenarbeit mit Paneth auf einem der Hauptarbeitsgebiete des Radiuminstituts liegt, nämlich der Aufklärung der verschiedenen Zerfallsreihen, und durch Paneths Übernahme einer Assistentenstelle an der Deutschen Technischen Hochschule in Prag 1917 ihr Ende findet, wird durch die Themenstellung der gemeinsamen Arbeiten Meyers und Przibrams dem Institut das Arbeitsfeld der Beeinflussung von Festkörpern durch Strahlen eröffnet. Przibram baut dieses Gebiet ab Mitte der Zwanzigerjahre mit einer eigenen Arbeitsgruppe als späterer Vizedirektor des Radiuminstituts zu einem sehr erfolgreichen Teilbereich der Aktivitäten des Radiuminstituts weiter aus.

Die mit Otto Hahn (zweiter Koautor E. v. Schweidler) gemeinsam verfaßte Arbeit bezieht sich auf den Bericht über die Versammlung der internationalen Radiumstandardkommission vom März 1912 in Paris (Nr. 78 und 78a).

Größere zusammenfassende Arbeiten in Form von Übersichts- und Handbuchartikel verfaßte Meyer ab der Mitte der Zwanzigerjahre bis zur Mitte der Dreißigerjahre. Neben dem Übersichtsartikel „Die radioaktiven Substanzen“ im „Handbuch für biologische Arbeitsmethoden“ und der Arbeit 
„Eichungs- und Meßmethoden für radioaktive Substanzen“ im „Handbuch der gesamten Strahlenheilkunde, Biologie, Pathologie und Therapie“, die sich beide an ein nicht primär physikalisch ausgebildetes Publikum wenden, schreibt Meyer 1923 den Artikel „Radioaktive Substanzen“ für das Tabellenwerk „Landolt-Börnstein“ mit den nachfolgenden Ergänzungen 1927, 1930, 1935, sowie 1926 den Artikel im „Handbuch der Physik“ („Geiger-Scheel“) zum Thema „Die radioaktiven Stoffe“ (2. Auflage 1933).

Abschliessend sei noch festgehalten, daß Meyer nur bei solchen Publikationen als Autor bzw. Koautor aufscheint, bei welchen sein unmittelbarer und direkter Anteil an den Arbeiten dies rechtfertigte. Meyer lehnte es entschieden ab, lediglich als Anreger einer Arbeit oder als Direktor des Instituts für sich Koautorschaft zu reklamieren.

Mit Bezug auf die Produktion der wissenschaftlichen Arbeiten Stefan Meyers werden fünf Arbeitsphasen unterschieden. (Siehe dazu die Abbildungen 1 und 2.)

Phase I (1896-1909): Frühphase der Radioaktivitätsforschung. Arbeiten zum Magnetismus. In dieser Zeit entstehen die wichtigen Arbeiten zum Verhalten der radioaktiven Strahlen im magnetischen Feld, sowie (ab 1904) die zehn Arbeiten „Untersuchungen über radioaktive Substanzen“.

Phase II (1910-1920): Erste Phase der Institutsaktivitäten und Vollendung der Frühphase der Radioaktivitätsforschung. Der 1. Weltkrieg 19141918 zeigt erst gegen sein Ende (1917/18) einen schwach erkennbaren Einfluß auf die Gesamtproduktivität; dieser Trend in der Gesamtproduktivität der Arbeiten (ab 1918) bleibt dann während der zwanziger Jahre bis in die dreißiger Jahre erhalten, sodaß kein Zusammenhang mit den Kriegsereignissen vermutet werden kann.

Das Meyer charakterisierende CEuvre entsteht in der Zeit zwischen 1896 und 1916/18 und findet seinen Abschluß in dem mit E. v. Schweidler gemeinsam publizierten Standardwerk „Radioaktivität“ (1916).

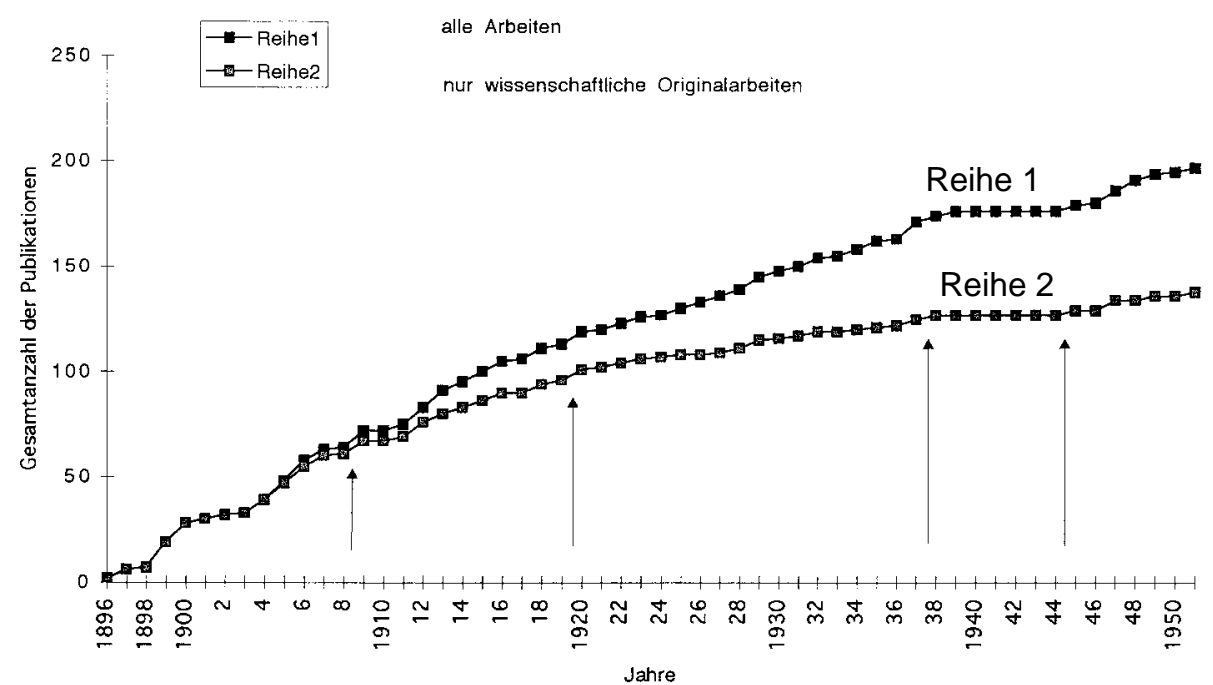

Abb. 1: Hier ist die Anzahl der im Publikationsverzeichnis aufgenommenen Arbeiten im zeitlichen Verlauf dargestellt. Reihe 1 zeigt alle Arbeiten, Reihe 2 nur die wissenschaftlichen Originalarbeiten (d. h. Zeitungsartikel, Nachrufe, Handbuch- und Übersichtsartikel u. ä. wurden bei der Zählung weggelassen). 


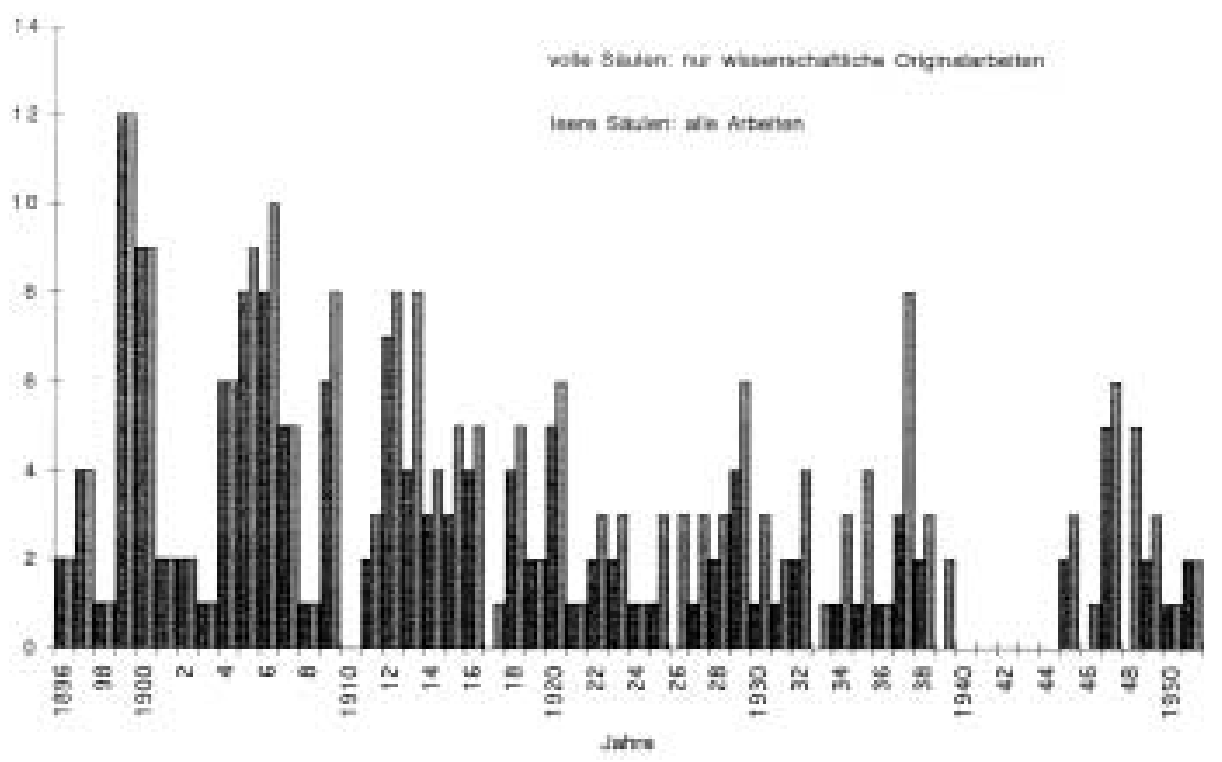

Abb. 2: Anzahl der wissenschaftlichen Publikationen pro Jahr im zeitlichen Verlauf zwischen 1896 und 1951. Zu den beiden Reihen (volle und leere Säulen) siehe auch Abbildung 1.

Im Zusammenhang mit der Ermittlung des Rangs emigrierter Physiker bzw. ihrer Bedeutung im jeweiligen Fach und die Zeit ihrer stärksten Wirkung stellte K. Fischer gemessen an den Zitationsziffern und bezogen auf die Periode 1920-1925 für St. Meyer „Rang 11“ der in dieser Periode meist zitierten Autoren im Fach Kernphysik fest. Die Ränge 1 bis 10 nehmen Bohr, Fajans, Born, Coster, Langmuir, Rutherford, Richardson, Hevesy, Franck und Baxter ein; die Ränge 12 und 13 Geiger und Ladenburg. In der folgenden Periode 1926-1930, die von Rutherford und Chadwick angeführt wird, erscheint Meyer nicht mehr unter den ersten 20 Rängen. Dieser zitationsanalytische Befund stützt die Aussage über das spätestens 1920 in Bezug auf die intradisziplinäre Wirkung abgeschlossene Cuvre Stefan Meyers. (Vgl. dazu Seite 117 und Fußnote 30.)

Phase III (1921-1938): Phase der reduzierten kontinuierlichen Produktion im Gesamtgebiet der Radioaktivität und Kernphysik, besonders auch im Bereich der medizinisch-balneologischen Anwendungen. Phase der „Mentorschaft".

Phase IV (1939-1945): Phase des erzwungenen Schweigens während der Zeit des Nationalsozialismus in Österreich.

Phase V (1945/46-1951): Spätphase; neben einigen Originalarbeiten mehrere historisch-reflexive Publikationen; zwei kunsthistorische Skizzen. Weiters werden jene Arbeiten publiziert, die während der Jahre 1938-1945 als „Zwangspensionist“ in Bad Ischl entstanden. 


\title{
Stefan Meyer
}

\author{
Gesamtverzeichnis der Schriften
}

1896

1. Über den Sitz der Potentialdifferenzen in Tropfelektroden und im Capillarelektrometer. Wiener Sitz. ber. CV. IIa, 139-160, 1896

2. Über die Fortpflanzungsgeschwindigkeit eines mechanischen Impulses in gespannten Drähten. Wiener Sitz. ber. CV. IIa, 1015-1024, 1896

\section{7}

3. Bestimmung der Magnetisirungszahlen von Flüssigkeiten und deren Änderung mit der Temperatur. (I. Mittheilung.) (gem. mit Gustav Jäger). Wiener Sitz. ber. CVI. IIa, 594-623, 1897

4. Bestimmung der Magnetisirungszahlen von Flüssigkeiten und deren Änderung mit der Temperatur. (II. Mittheilung.) (gem. mit Gustav Jäger). Wiener Sitz. ber. CVI. IIa, 623-654, 1897

5. Über Atommagnetismus. Verh. d. Ges. Dt. Naturf. und Ärzte, Braunschweig, 47-48, 1897

6. Über magnetische Susceptibilität und Atommagnetismus. (gem. mit Gustav Jäger). Wiedemann Ann. d. Phys. u. Chem. 63, 83-90, 1897

\section{8}

7. Bestimmung der Magnetisirungszahlen von Flüssigkeiten und deren Änderung mit der Temperatur. (III. Mittheilung.) (gem. mit Gustav Jäger). Wiener Sitz. ber. CVII. IIa, 5-13, 1898

1899

8. Über die Magnetisirungszahl des Wassers. (gem. mit Gustav Jäger). Wiedemann Ann. d. Phys. u. Chem. 67, 427-429, 1899

9. Die Magnetisirungszahl des Wassers. (gem. mit Gustav Jäger). Wiedemann Ann. d. Phys. u. Chem. 67, 707-713, 1899

10. Die magnetische Susceptibilität des Wassers. (gem. mit Gustav Jäger) Wiener Sitz. ber. CVIII. IIa, 261-272, 1899

11. Über die magnetischen Eigenschaften der Elemente. Wiener Sitz. ber. CVIII. IIa, 171-184, 1899

11a. Über die magnetischen Eigenschaften der Elemente. Wiedemann Ann. d. Phys. u. Chem. 68., 325-334, 1899

12. Über Krystallisation im magnetischen Felde. (I. Mittheilung.) Wiener Sitz. ber. CVIII. IIa, 513-515, 1899

13. Volumenometrische Bestimmung des specifischen Gewichtes von Yttrium, Zirkonium und Erbium. Wiener Sitz. ber. CVIII. IIa, 767-770, 1899

14. Magnetisirungszahlen anorganischer Verbindungen. Wiener Sitz. ber. CVIII. II a, 861-898, 1899 
14a. Magnetisirungszahlen anorganischer Verbindungen. Wiedemann Ann. d. Phys. u. Chem. 69., 236-263, 1899

15. Über das Verhalten von Radium und Polonium im magnetischen Felde. (gem. mit E. v. Schweidler) Phys. Zts. I., 90-91, 1899

15a. Über das Verhalten von Radium und Polonium im magnetischen Felde. (gem. mit E. v. Schweidler) Wiener Anzeiger XXII., 3. Nov. 1899

16. Über das Verhalten von Radium im magnetischen Felde.(gem. mit E. v. Schweidler) Wiener Anzeiger XXIII., 9. Nov. 1899

17. Über das Verhalten von Radium und Polonium im magnetischen Felde. (II. Mitt.) (gem. mit E. v. Schweidler) Phys. Zts. I., 113-114, 1899

18. Versuche über die Absorption von Radiumstrahlen. (gem. mit E. v. Schweidler) Wiener Anzeiger XXVI., 7. Dez. 1899

19. Bemerkung zu der Abhandlung der Herren H. du Bois und O. Liebknecht: Moleculare Susceptibilität der Salze seltener Erden. Verh. d. Dt. Phys. Ges. I., 275-277, 1899

\section{0}

20. Versuche über die Absorption von Radiumstrahlen. (gem. mit E. v. Schweidler) Phys. Zts. I., 209-211, 1900

21. Über Radium- und Poloniumstrahlung. (gem. mit E. v. Schweidler) Wiener Sitz. ber. CIX. IIa, 92-102, 1900

22. Bemerkung zu der Abhandlung der Herren H. du Bois und O. Liebknecht: Molekulare Susceptibilität seltener Erden. Ber. d. Dt. chem. Ges. XXXIII., 320-321, 1900

23. Weitere Beobachtungen an Becquerel-Strahlen. (gem. mit E. v. Schweidler) Wiener Anzeiger VII., 1. März 1900

24. Bestimmung einiger Magnetisierungszahlen. Wiener Sitz. ber. CIX IIa, 400-404, 1900

24a. Bestimmung einiger Magnetisirungszahlen. Ann. d. Phys. (IV) 1., 664667,1900

25. Über Atommagnetismus und Molecularmagnetismus. Wiener Sitz. ber. CIX IIa., 284-289, 1900

25a. Über Atommagnetismus und Molecularmagnetismus. Ann. d. Phys. (IV) $1 ., 668-672$

26. Über die Additivität der Atomwärmen. Wiener Sitz. ber. CIX. IIa, 405410,1900

26a. Über die Additivität der Atomwärmen. Ann. d. Phys. (IV) 2., 135-140, 1990

27. Über magnetische Susceptibilität und Atomvolumen. Phys. Zts. I., 40, 433-434, 1900

28. Über die Additivität von Atomeigenschaften. Ber. d. Dt. chem. Ges. XXXIII., 12, 1918-1919, 1900

\section{1}

29. Magnetisierungszahlen seltener Erden. Wiener Sitz. ber. CX. IIa, 541559,1901

30. Bemerkung zu der Abhandlung des Hrn. J. Koenigsberger betreffs der Susceptibilität des Wassers. (gem. mit Gustav Jäger) Ann. d. Phys. (IV) 6., 870,1901 
31. Notiz über das magnetische Verhalten von Europium, Samarium und Gadolinium. Wiener Sitz. ber. CXI. IIa, 38-41, 1902

32. Über die durch den Verlauf der Zweiphasencurve bedingte maximale Arbeit. Wiener Sitz. ber. CXI. IIa, 305-310, 1902

32a. Über die durch den Verlauf der Sättigungscurve bedingte maximale Arbeit. Ann. d. Phys. (IV) 7., 937-941, 1902

\section{3}

33. Über das Wachstum der Kristalle. (Vortrag, gehalten den 7. Jänner 1903) Ver. z. Verbreitung naturw. Kennt., XLIII, 6, 1903

\section{4}

34. Über Magnetisierung durch Tonanregung. Boltzmann-Festschrift. 6870, 1904

Redaktion der Boltzmann-Festschrift

35. Über den Einfluß von Temperaturänderungen auf radioaktive Substanzen. (Vorläufige Mitteilung) (gem. mit E. v. Schweidler) Phys. Zts. 5., 12, 319-320, 1904

36. Untersuchungen über radioaktive Substanzen I. Über den Einfluß von Temperaturänderungen und über die durch Pechblende induzierte Aktivität. (gem. mit E. v. Schweidler) Wiener Sitz. ber. CXIII. IIa, 763-790, 1904

37. Magnetisierungszahlen einiger organischer Verbindungen und Bemerkungen über die Unabhängigkeit schwach magnetischer Flüssigkeiten von Feldstärke und Dissoziation. Wiener Sitz. ber. CXIII. IIa, 1007-1017, 1904

38. Untersuchungen über radioaktive Substanzen II. Über die Strahlung des Uran. (gem. mit E. v. Schweidler) Wiener Sitz. ber. CXIII. IIa, 1057-1079, 1904

39. Untersuchungen über radioaktive Substanzen III. Über zeitliche Änderungen der Aktivität. Vorläufige Mitteilung. (gem. mit E. v. Schweidler) Wiener Anzeiger XXV., 1. Dez. 1904

\section{5}

40. Bericht über die Untersuchung der Vöslauer Therme auf Radioaktivität. (gem. mit H. Mache) Selbstverlag der Gemeinde 1-4, Vöslau, 1905

41. Ü̉ber die radioaktiven Substanzen. Österr. Rundschau II., 9-21, 1905

42. Eine Methode zur quantitativen Untersuchung radiumhältiger Substanzen. (gem. mit H. Mache und E. v. Schweidler) Wiener Anzeiger VI., 16. Feb. 1905

43. Untersuchungen über radioaktive Substanzen (III. Mitteilung) Über zeitliche Änderungen der Aktivität. (gem. mit E. v. Schweidler) Wiener Sitz. ber. CXIV. IIa, 387-395, 1905

44. Über die Radioaktivität der böhmischen Bädergruppe: Karlsbad, Marienbad, Teplitz-Schönau-Dux, Franzensbad sowie von St. Joachimsthal. (gem. mit H. Mache) Wiener Sitz. ber. CXIV. IIa, 355-385, 1905

45. Über die Radioaktivität einiger Quellen der südlichen Wiener Thermenlinie. (gem. mit H. Mache) Wiener Sitz. ber. CXIV. IIa, 545-551, 1905 
46. Untersuchungen über radioaktive Substanzen. (IV. Mitteilung.) Zur Kenntnis des Aktiniums. (gem. mit E. v. Schweidler) Wiener Sitz. ber. CXIV. IIa, 1147-1158, 1905

47. Untersuchungen über radioaktive Substanzen. V. Mitteilung: Über Radioblei und Radium-Restaktivitäten. (gem. mit E. v. Schweidler) Wiener Sitz. ber. CXIV. IIa, 1195-1219, 1905

48. Über die Radioaktivität österreichischer Thermen. (gem mit H. Mache) Phys. Zts. 6., 692-700, 1905

48a. Über die Radioaktivität oesterreichischer Thermen. Premier Congrès Internat. pour l'Étude de la Radiologie et de l'Ionisation. Liége, September 1905. 18-37, 1905

\section{6}

49. Untersuchungen über radioaktive Substanzen. (VI. Mitteilung.) Über Radium F (Polonium). (gem. mit E. v. Schweidler) Wiener Sitz. ber. CXV. IIa, 63-88, 1906

50. Bemerkung zu der Mitteilung der Frau Sklodowska Curie „Über die Zeitkonstante des Poloniums; Nachtrag". (gem. mit E. v. Schweidler) Phys. Zts. 7., No. 8, 257-258, 1906

51. Über die neueren Ergebnisse und die Methoden der radioaktiven Forschung (Vortrag vom 23. Jan. 1906). Vierteljahresbericht d. Wr. Ver. z. Förd. d. phys. u. chem. Unterr. XI., 1, 1906

51a. Die neueren Ergebnisse und die Methoden der radioaktiven Forschung. Wissen f. Alle, 323-325, 1906

52. Untersuchungen über radioaktive Substanzen. VII. Über die aktiven Bestandteile des Radioblei. (vorläufige Mitteilung) (gem. mit E. v. Schweidler) Wiener Anzeiger XII., 26. April, 161-166, 1906

53. Untersuchungen über radioaktive Substanzen. VIII. Über ein radioaktives Produkt aus dem Aktinium. Vorläufige Mitteilung (gem. mit E. v. Schweidler) Wiener Anzeiger XII, 26. April, 166-169, 1906

54. Untersuchungen über radioaktive Substanzen. (VII. Mitteilung.) Über die aktiven Bestandteile des Radiobleis. (gem. mit E. v. Schweidler) Wiener Sitz. ber. CXV. IIa, 697-711, 1906

55. Untersuchungen über radioaktive Substanzen.(IX. Mitteilung.) Einige Versuche über die Absorption der $\alpha$-Strahlung in Aluminium. (gem. mit E. v. Schweidler) Wiener Sitz. ber. CXV. IIa, 713-738, 1906

56. Zur Erinnerung an Ludwig Boltzmann. Österr. Rundschau, 25. X., 479483,1906

57. Über das Zerschlagen alter Glühlampen. Phys. Zts. 7., No. 24, 912, 1906

58. Zur Charakteristik der activen Bestandtheile des Radiobleies. (gem. mit E. v. Schweidler) Lieben-Festschrift, 402-409, 1906

\section{7}

59. Bericht über die Endglieder der Radiumzerfallsreihe (Radioblei). (gem. mit E. v. Schweidler) Jahrb. d. Rad. u. Elektr. 3., 381-397, 1907

59a. Nachtrag zu unserem Bericht über die Endglieder der Radiumzerfallsreihe (Radioblei). (gem. mit E. v. Schweidler) Jahrb. d. Rad. u. Elektr. 4., 112-113, 1907

60. Bemerkungen zum periodischen System der Elemente und Versuch der Einordnung der Radioelemente in dasselbe. (Vortrag, gehalten am 
11. Dezember 1906) Vierteljahresbericht d. Wr. Ver. z. Förd. d. phys. u. chem. Unterr. 12., 8-20, 1907

61. Untersuchungen über radioaktive Substanzen. (VIII. Mitteilung). Über ein radioaktives Produkt aus dem Aktinium. (gem. mit E. v. Schweidler) Wiener Sitz. ber. CXVI. IIa, 315-322, 1907

62. Untersuchungen über radioaktive Substanzen. (X. Mitteilung). Über die Zerfallskonstante von Radium D. (gem. mit E. v. Schweidler) Wiener Sitz. ber. CXVI. IIa, 701-713, 1907

62a. Über die Zerfallskonstante von Radium D. (gem. mit E. v. Schweidler) Phys. Zts. 8., 457-461, 1907

63. Bemerkung zu einer Notiz des Herrn H. W. Schmidt betreffend RaE $\mathrm{E}_{1}$ und $\mathrm{RaE}_{2}$ (gem. mit E. v. Schweidler) Phys. Zts. 8., 457, 1907

\section{8}

64. Zur Kenntnis der Magnetisierungszahlen seltener Erden. Wiener Sitz. ber. CXVII. IIa, 995-1000, 1908

\section{9}

65. Über radioaktive Substanzen. (Vortrag, gehalten am 20. Nov. 1908) Zts. d. Österr. Ingenieur- u. Architektenvereins Nr. 2 u. 3, 1909

65a. Über radioaktive Substanzen. Urania II. Nr. 2, 17-22, 1909

66. Die Radiumforschung in Österreich. Urania, II. Nr. 12, 184-186, 1909

67. Eine Verbesserung am Engler-Sievekingschen Apparat zur Bestimmung des Emanationsgehaltes von Quellwässern. (gem. mit H. Mache) Zts. f. Instrumentenkunde, 65-68, 1909

67a. Eine Verbesserung am Engler-Sievekingschen Apparat zur Bestimmung des Emanationsgehaltes von Quellwässern. (Vortrag und Demonstration in der Ausstellung der 81. Versammlung d. Dt. Naturforsch. u. Ärzte zu Salzburg am 23. September 1909) (gem. mit H. Mache) Phys. Zts. 10., 860-862, 1909

67b. Eine Verbesserung am Engler-Sievekingschen Apparat zur Bestimmung des Emanationsgehaltes von Quellwässern. (Vortrag und Demonstration in der Ausstellung der 81. Versammlung d. Dt. Naturforsch. u. Ärzte zu Salzburg am 23. September 1909) (gem. mit H. Mache) Verh. d. Dt. Phys. Ges. Nr. 20, 519-522, 1909

68. Observations on the periodic system of the elements, and an attempt at classifying the radioelements in the same. Ion. I., 4., 249-259, 1909

69. On the change of colour of Kunzite by Becquerel-Radiation. Ion. I. 4., 260, 1909

70. Über das Vorkommen von Ionium in den Rückständen der Pechblende. (gem. mit E. v. Schweidler) Wiener Anzeiger Nr. XIV, 11. Juni 1909

71. Über das Verhalten von Kunzit unter Einwirkung von Becquerelstrahlung. Phys. Zts. 10., 483-484, 1909

72. Bericht über das Uran und seine ersten Zerfallsprodukte. Jahrb. d. Rad. u. Elektr. 4., 381-408, 1909

1911

73. Über die Erreichung der Sättigungswerte bei Ionisation durch $\alpha$-Strahlen. (gem. mit V. F. Hess) (MIR Nr. 2) Wiener Sitz. ber. CXX. IIa, 11871198,1911 
74. Das Wiener Radium-Institut. (aus einem Gespräch) Umschau, 7. Jan. 1911, Nr. 2, 26-29, 1911

75. Über Radium und Mesothor aus Monazitsand. (gem mit L. Haitinger und K. Peters) (radiologischer Teil von St. Meyer) (MIR Nr. 3) Wiener Sitz. ber. CXX. IIa, 1199-1204, 1911

\section{2}

76. Über Radiumnormale. (gem. mit H. Mache) Phys. Zts. 13., 320-322, 1912

77. Zur Definition der Wiener Radiumstandardpräparate. (gem. mit V. F. Hess) (MIR Nr. 17) Wiener Sitz. ber. CXXI. IIa, 603-631, 1912

78. Bericht über die Versammlung der internationalen Radiumstandardkommission in Paris vom 25. bis 28. März 1912. (gem. mit O. Hahn und E. v. Schweidler) Phys. Zts., 13. 524-525, 1912

78a. Bericht über die Versammlung der internationalen Radiumstandardkommission in Paris vom 25. bis 28. März 1912. (gem. mit O. Hahn und E. v. Schweidler) Rad. in Biologie und Heilkunde 1., 354-356, 1912

79. Notiz über die Maßeinheiten der Radiumemanation. (gem. mit H. Mache) Rad. in Biologie und Heilkunde I., 350-353, 1912

80. Über die Intensität der $\alpha$-Strahlung von Uran. (gem. mit F. Paneth) (MIR Nr. 23) Wiener Sitz. ber. CXXI. IIa, 1403-1412, 1912

81. Über einige neue Erscheinungen bei der Beeinflussung von Gläsern und Mineralien durch Becquerelstrahlung. (gem. mit K. Przibram) (MIR Nr. 24) Wiener Sitz. ber. CXXI. IIa, 1413-1418, 1912

82. Zur „Notiz über die Maßeinheiten der Radiumemanation“. (gem. mit H. Mache) Rad. in Biologie und Heilkunde II., 96-98, 1912

83. Zur Erinnerung an Werner v. Bolton. Neues Wiener Tagblatt, 27. Dez. 1912

\section{3}

84. Maße und Meßmethoden. (gem. mit H. Mache) Handbuch der RadiumBiologie und -Therapie (Hrsg. v. P Lazarus), J. F. Bergmann, Wiesbaden, $59-74,1913$

85. Notiz zu dem neuesten Wert für das Atomgewicht des Radiums. Phys. Zts. 14., 124-125, 1913

86. Über die Normalmaße des Radiums und die Meßmethodik der Radioaktivität. Zts. f. Balneol., Klimatol. u. Kurorthygiene 6., Nr. 2, 1913

86a. Über die Normalmasse der Radiums und die Meßmethodik der Radioaktivität. Strahlentherapie II., 2., 533-542, 1913

87. Radium-Normalmasse. Umschau Nr. 17., 187-190, 1913

88. Über die Lebensdauer von Uran und Radium. (MIR Nr. 48) Wiener Sitz. ber. CXXII. IIa, 1085-1094, 1913

89. Bemerkungen über die Löslichkeit von Radiumemanation und anderen Gasen in Flüssigkeiten. (MIR Nr. 50) Wiener Sitz. ber. CXXII. IIa, 12811294,1913

90. Über die im Wiener Radiuminstitute vorgenommene Herstellung einiger sekundärer Radiumetalons. Wiener Anzeiger, Nr. XXV, 4. Dez. 1913

91. Die Bedeutung der Radioaktivität für die Mineralogie. In: Handbuch der Mineralchemie (Hrsg. v. C. Doelter) III. 2., 235-248, 1913 
92. Über die Verfärbung von Salzen durch Becquerelstrahlen und verwandte Erscheinungen (gem. mit K. Przibram) (MIR Nr. 58) Wiener Sitz. ber. CXXIII. IIa, 653-663, 1914

93. Radioaktive Strahlungen und Umwandlungen. (gem. mit E. v. Schweidler) Kultur d. Gegenwart (Hrsg. v. P. Hinneberg), Physik (unter Redaktion v. E. Warburg), 495-513, 1914

94. $\gamma$-Strahlenmessung von Mesothorpräparaten. (gem. mit V. F. Hess) (MIR Nr. 62) Wiener Sitz. ber. CXXIII. IIa, 1443-1458, 1914

95. Neue Reichweitenbestimmungen an Polonium, Ionium und Actiniumpräparaten. (gem. mit V. F. Hess und F. Paneth) (MIR Nr. 64) Wiener Sitz. ber. CXXIII. IIa, 1459-1488, 1914

\section{5}

96. Über die Maßeinheiten bei Gehaltsbestimmungen radioaktiver Substanzen. Jahrb. d. Rad. u. Elektr. 11. Heft, 4., 436-450, 1915

97. Wilhelm Michl (Wien). Nachruf. Phys. Zts. 16., 69-70, 1915

98. Magnetisierungszahlen isotoper Stoffe. (MIR Nr. 77) Wiener Sitz. ber. 124., IIa, 187-192, 1915

99. Über die Atomvolumenkurve und über den Zusammenhang zwischen Atomvolumen und Radioaktivität. (MIR Nr. 78) Wiener Sitz. ber. 124. IIa, 249-262, 1915

99a. Einige Bemerkungen über Atomeigenschaften. Sonderdruck aus Arbeiten aus den Gebieten der Physik, Mathematik, Chemie. Julius Elster und Hans Geitel gewidmet. 146-160, 1915

100. Friedrich Hasenöhrl. Nachruf. Phys. Zts. 16., 429-433, 1915

\section{6}

101. Die Lebensdauer des Ioniums und einige Folgerungen aus der Bestimmung dieser Konstante. (MIR Nr. 88) Wiener Sitz. ber. 125. IIa, 191-199, 1916

102. Über die Beziehung zwischen Zerfallskonstanten und Reichweiten. (MIR Nr. 89) Wiener Sitz. ber. 125. IIa, 201-207, 1916

103. Zur Kenntnis der mittleren Lebensdauer des Radiums. (gem mit R. W. Lawson) (MIR Nr. 94) Wiener Sitz. ber. 125. IIa, 723-733, 1916

104. Notiz über die Zerfallskonstante des Radiothor. (gem mit F. Paneth) (MIR Nr. 96) Wiener Sitz. ber. 125. IIa, 1253-1260, 1916

105. Radioaktivität (gem. mit E. v. Schweidler) Naturwissenschaft und Technik in Lehre und Forschung (hrsg. von K. T. Fischer). Verlag von B. G. Teubner, Leipzig und Berlin 1916

1917

106. Über Atome, Elektronen, Quanten. (Vortrag, gehalten am 17. März 1917) Vierteljahresber. d. Wr. Ver. z. Förd. d. phys. chem. Unterr. Heft 1, 1917

\section{8}

107. Die Nomenklatur der Radioelemente (gem. mit E. v. Schweidler) Zts. f. Elektrochem. 24, 36-38, 1918 
107a.Die Nomenklatur der Radioelemente (gem. mit E. v. Schweidler) Phys. Zts. 19., 30-32, 1918

108. Periodische Systeme der Elemente. Phys. Zts. 19., 178-179, 1918

109. Neuere Ergebnisse der radioaktiven Forschung. (Vortrag, gehalten am 13. Feb. 1918). Ver. z. Verbreitung naturw. Kenntn. 58., Heft 7, 1918

110. Über die Actinium-Zerfallsprodukte. (gem mit F. Paneth) (MIR Nr. 104) Wiener Sitz. ber. 127. IIa, 147-193, 1918

111. Zur Frage nach der Existenz von Isotopen mit gleichem Atomgewicht. Die Endprodukte der Thoriumzerfallsreihe. (MIR Nr. 111) Wiener Sitz. ber. 127. IIa, 1283-1296, 1918

\section{9}

112. Thor- und Urangehalt einiger Erze, nebst Anhang: Über die zeitliche Änderung von ThB-ThC. (MIR Nr. 121) Wiener Sitz. ber. 128. IIa, 897-908, 1919

113. Über die Konstanz des Verhältnisses von Actinium zu Uran in natürlichen Erzen. (gem. mit V. F. Hess) (MIR Nr. 122) Wiener Sitz. ber. 128. IIa, 909-924, 1919

\section{0}

114. Über den Zusammenhang einiger physikalischer Eigenschaften mit der Elektronenordnung in den Elementen. Naturwissenschaften 8, 284, 1920

115. Wie groß ist der Mond? Phys. Zts. 21., 168, 1920

116. Zur Kenntnis der Zerfallskonstante des Actinium und des Abzweigungsverhältnisses der Actiniumreihe. (MIR Nr. 130) Wiener Sitz. ber. 129. IIa, 483-490, 1920

117. Zur Frage nach der Existenz von Isotopen mit gleichem Atomgewicht. Zts. f. phys. Chemie 95., 407-433, 1920

118. Das erste Jahrzehnt des Wiener Instituts für Radiumforschung. Jahrb. d. Rad. u. Elektr. 17., 1-29, 1920

119. Radioaktive Konstanten nach dem Stand von 1920. Jahrb. d. Rad. u. Elektr. 17., 80-87, 1920

\section{1}

120. Messung der Radioaktivität der Teplitzer Thermen. (publ. von E. Stein) Wr. Med. Wochenschr. Nr. 12, 20, 22, 1921

\section{2}

121. Über Verfärbung und Lumineszenz durch Becquerelstrahlen. (gem. mit K. Przibram) Zts. f. phys. Chemie 100., 334-336, 1922

122. Bemerkungen über Verfärbung und Lumineszenz unter Einwirkung von Becquerelstrahlen. (gem. mit K. Przibram) (MIR Nr. 147) Wiener Sitz. ber. 131. IIa, 429-435, 1922

123. Radioaktivität. 16 Manuskriptseiten, 4 Textfiguren; abgeschickt an Prof. Auerbach, Jena, 6. Dez. 1922 
124. Radioaktive Konstanten. In: W. A. Roth - K. Scheel, Konstanten der Atomphysik (Landolt-Börnstein, 5. Aufl.) 12-23, 1923

125. Radioaktive Konstanten nach dem Stande von 1923. Jahrb. d. Rad. u. Elektr. 19. 334-344, 1923

126. Über den Gehalt von Ionium-Thorium in der Uranpechblende von St. Joachimsthal. (gem. mit C. Ulrich) (MIR Nr. 158) Wiener Sitz. ber. 132. IIa, 79-84, 1923

\section{4}

127. Magnetisierungszahlen seltener Erden. (MIR Nr. 171) Wiener Sitz. ber. 134. IIa, 491-496, 1924

\section{5}

127a.Magnetisierungszahlen seltener Erden. Phys. Zts. 26., 51-54, 1925

128. Entdeckungsgeschichte und Grundtatsachen der Radioaktivität (von J. Elster und H. Geitel). (überarbeitete Fassung für die 2. Auflage; gem mit E. v. Schweidler) Kultur d. Gegenwart. 2. Auflage, Bd. Physik, 521-540, 1925

129. Radioaktive Strahlungen und Umwandlungen. (gem mit E. v. Schweidler) Kultur d. Gegenwart. 2. Auflage, Bd. Physik, 541-560, 1925

130. Bemerkungen zu den Magnetisierungszahlen seltener Erden. Phys. Zts. 26., 478-479, 1925

\section{6}

131. Die radioaktiven Stoffe. In: Handbuch der Physik (Geiger-Scheel) Bd. XXII, 222-277, 1926

132. Neuere Erkenntnisse über den Aufbau der Atome. (Vortrag, gehalten am 24. März 1926) Ver. z. Verbreitung naturw. Kennt. 66., 61-79, 1926

133. Die radioaktiven Substanzen. In: Handb. d. biol. Arbeitsmethoden (Hrsg. von E. Abderhalden) Wien: Urban u. Schwarzenberg (1926). II, 2., 15371643

\section{7}

105a.Radioaktivität (gem. mit E. v. Schweidler) 2., vermehrte und teilweise umgearbeitete Auflage. Verlag von B. G. Teubner, Leipzig und Berlin 1927

134. Radioaktive Konstanten. In: W. A. Roth - K. Scheel, Konstanten der Atomphysik (Landolt-Börnstein) Ergänzungsbd. 1927

135. Bemerkungen über Atomgewichte und Packungseffekte. Naturwissenschaften 15., 623-624, 1927

136. Eichungs- und Meßmethoden für radioaktive Substanzen. In: Handb. d. ges. Strahlenheilkunde, Biologie, Pathologie und Therapie (Hrsg. v. P. Lazarus) 167-177, 1927

137. Über die Zerfallskonstante des Actiniums. (MIR Nr. 218) Wiener Sitz. ber. 137. IIa, 235-239, 1928 
138. Über die Bausteine der Atomkerne. Scientia 89., 98, Aug. 1928

139. Bemerkungen zu den Beziehungen unter den Atomgewichten von Uran, Radium, RaG und Helium. (MIR Nr. 226) Wiener Sitz. ber. 137. IIa, 599606,1928

\section{9}

140. Zur Frage nach der Bildung von Neutronen. (MIR Nr. 229a) Wiener Anzeiger Nr. 11, 18. April 1929, 66., 101-104, 1929

141. Zur Darstellung der Packungseffekte der Atome. (MIR Nr. 235) Wiener Sitz. ber. 138. IIa, 431-438, 1929

142. Physikalische Grundlagen der Radiumemanationstherapie. (MIR Nr. 238) Wiener Sitz. ber. 138. IIa, 557-580, 1929

143. Physikalische Beiträge zur Radiumemanationstherapie. (gem. mit H. Mache) Abh. aus d. Gesamtgebiet d. Hygiene (Hrsg. von R. Graßberger) Heft 5, 1929

144. Bericht über die Untersuchung der Quellen von Warmbad Villach auf ihren Gehalt an Radium-Emanation (Manuskript) Dez. 1929

145. Neuere Anschauungen über das Werden und Vergehen der Materie. (Manuskript) Vortrag, gehalten am 3. Juni 1929 in der Gesellschaft für Geophysik und im Gauverein Österreich der Physikalischen Gesellschaft Wien

\section{0}

142a.Physikalische Grundlagen der Radiumemanationstherapie. Forsch. u. Fortsch. 6., 150, 1930

146. Radioaktive Konstanten. In: W. A. Roth - K. Scheel, Konstanten der Atomphysik (Landolt-Börnstein) 2. Ergänzungsbd. 1930

147. Zur Verwendung der Radiumemanation als Indikator für Diagnostik und Therapie. Untersuchungen an Atmungsvorgängen. (gem. mit E. Suess) (MIR Nr. 269) Wiener Sitz. ber. 139. IIa, 613-628, 1930

148. Haitingerfeier. Ansprache. Österr. Chemiker-Zeitung 33., 181-182, 1. Nov. 1930

\section{1}

149. Verwendungsweise radioaktiver Präparate. Medizin. Welt 5., Nr. 29, 1027-1032, 1931

150. Die radioaktiven Konstanten nach dem Stand von 1930. Bericht der Intern. Radium-Standard-Kommission. Phys. Zts., 32., 569-581, 1931

\section{2}

151. Protonenzahlen, Kernladungszahlen und Reichweiten von $\alpha-$ Strahlern. (MIR Nr. 288) Wiener Sitz. ber. 141. IIa, 71-78, 1932

152. Zur Wahl der Basis für die Atomgewichte. Phys. Zts. 33., 301-302, 1932

153. Entwicklungsrichtungen der radioaktiven Forschung und Auswirkungen auf Nachbargebiete. (Vortrag, gehalten bei der Bunsen-Gesellschaft in Münster, 1932) Zts. f. Elektrochem. 38, 480-488, 1932

154. Physikalische Grundlagen der Radium-Emanations-Therapie. Pharmazeut. Presse, Dez. 1932, 1-6, 1932 
155. Die radioaktiven Stoffe. In: Handb. d. Physik (Hrsg. von H. Geiger und K. Scheel) XXII. (1. Teil) (2. Aufl.) 245-310, 1933

\section{4}

156. Zur Beziehung zwischen Anfangsgeschwindigkeit und Reichweite der Alphateilchen. (MIR Nr. 340a) Wiener Anzeiger Nr. 18, 5. Juli 1934, 71., 207-208

157. Madam Curie. Nachruf. Naturwissenschaften 22., 517-518, 1934

157a.Mevrouw Curie. Wetenschapenlijke Bladen, Haarlem 247-250, 1934

158. Radiumemanation und ihre medizinische Verwendung. Vortrag 10. 1. 1934. Ver. z. Verbreitung naturw. Kennt., 1934

159. Radioaktive Konstanten. In: W. A. Roth - K. Scheel, Konstanten der Atomphysik (Landolt-Börnstein) 3. Ergänzungsbd. 1935

160. Einige Grundlagen für die Radiumemanationstherapie. Badgasteiner Badeblatt Nr. 9, 10 u. 11, 1935

161. Zur Berechnung der Geschwindigkeiten der Alphateilchen aus ihren Reichweiten und über die Beziehung zur Zahl der von ihnen erzeugten Ionenpaare. (MIR Nr. 362) Wiener Sitz. ber. 144. IIa, 317-330, 1935

162. 25 Jahre Radiuminstitut. Wiener Anzeiger Nr. 20. Sitz. ber. math. nat. Kl., 24. Okt. 1935

\section{6}

163. Zur Frage nach der Existenz der Thor-Poloniums. (MIR Nr. 388) Wiener Sitz. ber. 145. IIa, 577-582, 1936

1937

164. Über das „Alter“ der Sonne, über die Zerfallskonstante des Actinurans und über die Mengenverhältnisse Blei zu Thor zu Uran auf der Erde. (MIR Nr. 393) Wiener Sitz. ber. 146 IIa, 175-197, 1937

165. Die Lokomotive „Philadelphia“. Neues Wr. Tagblatt, 22. Mai 1937

166. Physikalische Grundlagen von Emanationskuren. Strahlentherapie 58., 656-663, 1937

167. Lord Rutherford of Nelson. Nachruf. Neues Wr. Tagblatt, 21. Okt. 1937

168. Further Tributes to the late Lord Rutherford. Nature, 140., 1047-1048, 1937

169. Über das „Alter“ der Sonne, über die Zerfallskonstante des Actinurans und über die Mengenverhältnisse Blei zu Uran auf der Erde. Naturwissenschaften 25., 764-765, 1937

170. Zur Erinnerung an Erhard Suess. Nachruf. Schola Hornana. 2. Heft, 15, 1937

171. Über das „Alter“ der Sonne, über die Zerfallskonstante des Actinurans. (II. Mitteilung) (MIR Nr. 407) Wiener Sitz. ber. 146 IIa, 581-588, 1937 
172. Über das „Alter“ der Sonne, über die Zerfallskonstante des Actinurans. Forsch. u. Fortsch. 14., 41-43, 1938

173. Bemerkungen zu den radioaktiven Konstanten von Uran und Radium. (MIR Nr. 411a) Wiener Anzeiger Nr. 3, 27. Jan. 1938, 75., 7-10

174. Eine einfache Formel zur Berechnung der Atomgewichte aus den Massenzahlen und Packeffekten. (MIR Nr. 420) Wiener Sitz. ber. 147. IIa, 249-260, 1938

\section{9}

175. Gustav Jäger. Nachruf. Almanach d. Wr. Akad. d. Wiss. für 1938. 88. 234-247, 1939

176. Lord Rutherford of Nelson. Nachruf. Almanach der Wr. Akad. d. Wiss. für 1938. 88., 251-262, 1939

\section{5}

177. Über die Radium-Standard-Präparate. (MIR Nr. 452a) Wiener Anzeiger 82. 25-30, 29. Nov. und 13. Dez. Nr. 8 u. 9, 1945

178. Zur Erinnerung an Otto Hönigschmid. Wiener Anzeiger Nr. 82, Nr. 8 u. 9, 29. Nov. und 13. Dez. 1945

179. Geschichte der Herstellung der primären Radium-Standards. (MIR Nr. 453a) Wiener Anzeiger Nr. 82, Nr. 8 u. 9, 29. Nov. und 13. Dez. 1945

\section{6}

180. Unsere Holzblasinstrumente. Von der Flöte zum Saxophon. (gem. mit Alexander Wunderer) Frohes Schaffen. 1946

180a.Musikinstrumente. In: Volkslexikon (Hg. Ed. Zellwecker) 1946/47

\section{7}

181. Zur Berechnung der Atomgewichte. Acta Phys. Austriaca I. 120-128, 1947

182. Bemerkung zur „Normalstimmung“. Acta Phys. Austriaca I. 129-130, 1947

183. Zur Genesis der chemischen Elemente. (gem. mit A. Koss-Rosenqvist) (MIR Nr. 456) Wiener Sitz. ber. 156. IIa, 625-640, 1947

183a.Zur Genesis der chemischen Elemente. (gem. mit A. Koss-Rosenqvist) Acta Phys. Austriaca II. 109-123, 1947

184. Kann man sich die Elemente mit durch die Zahl 4 teilbaren Atomgewichten aus Heliumpartikeln aufgebaut denken? (gem. mit A. Koss-Rosenqvist) (MIR Nr. 457) Wiener Sitz. ber. 156. IIa, 641-649, 1947

184a.Kann man sich die Elemente mit durch die Zahl 4 teilbaren Atomgewichten aus Heliumpartikeln aufgebaut denken? (gem. mit A. Koss-Rosenqvist) Acta Phys. Austriaca II. 124-132, 1947

185. Richtig Schauen. Für Maler, Konstrukteure, Photographen und Bilderfreunde. (Wien: Springer-Verlag, 1947)

186. Weitere Bemerkungen zum Schema der chemischen Elemente. (gem. mit A. Koss-Rosenqvist) Acta Phys. Austriaca II. 245-254, 1947 
187. Zur Erinnerung an die Jugendzeit der Radioaktivität. Lise Meitner (geb. 7. Nov. 1878) und Otto Hahn (geb. 8. März 1879) zu ihrem siebzigsten Geburtstag gewidmet. Naturwissenschaften 35., 161-163, 1948

188. Beiträge zur Kenntnis der atmosphärischen Elektrizität Nr. 78. Bericht über die Beobachtungen an der luftelektrischen Station in Seeham in den Sommern 1921-1942 von Egon Schweidler †. Hrsg. von Hans Benndorf und Stefan Meyer. Anzeiger d. Öst. Akad. d. Wiss. 4. Nov. 1948, Nr. 13, 195-209, 1948

189. Grundlagen der Instrumentenkunde für Musikanten und Dilettanten. (gem. mit Alexander Wunderer) (Wien: Universal Edition, 1948)

190. Hans Pettersson. Atlantis und Atlantik. Ins Deutsche übertragen von St. Meyer. (Wien: Springer-Verlag, 1948)

191. S. I. Wawilow: Isaac Newton (Buchbesprechung) Wr. Ztg. Nr. 211, Donnerstag, 9. Sept. 1948

\section{9}

192. Bemerkungen zum natürlichen Aufbau und zur Häufigkeitsverteilung der chemischen Elemente. Otto Hahn zum 70. Geburtstag zugeeignet. Zts. f. Naturforsch. 4a., 84-87, 1949

193. Zur Geschichte der Entdeckung der Natur der Becquerelstrahlen. Nach 50 Jahren. Naturwissenschaften 36., 129-132, 1949

194. Zum Problem des räumlich-plastischen Sehens. Anzeiger d. Öst.-Akad. d. Wiss. mathem. naturw. Kl. 13. Okt. 1949, Nr. 11, 209-212, 1949

195. Die Vorgeschichte der Gründung und das erste Jahrzehnt des Instituts für Radiumforschung. Festschrift des Instituts für Radiumforschung anläßlich seines 40jährigen Bestandes (1910-1950). Sitzungsberichte der mathem.-naturwiss. Kl. Abt. IIa, 159. Bd., 1.-2. Heft, 1950

1951

196. Der Dodo und der Aphanapterix. (Ein Bild von Roeland Savery) (als Manuskript fertiggestellt 1933) Anzeiger d. Öst. Akad. d. Wiss., phil.-hist. Kl., 4, 35-38, 1951

197. Über das Imhoff-Bildnis des Bartholomäus Bryn. (als Manuskript fertiggestellt 1933) Anzeiger d. Öst. Akad. d. Wiss., phil.-hist. Kl., 4, 38-41, 1951

\section{Bearbeitungen}

F. Lecher's Lehrbuch der Physik für Mediziner, Biologen und Psychologen. Bearbeitet von St. Meyer und E. v. Schweidler, Verlag von B. G. Teubner, Leipzig und Berlin

5. Auflage 1928

6. Auflage 1930

7. Auflage 1933

Nachdruck 1937

Nachdruck 8.-10-Auflage 1940 bis 1945

11. Auflage 1948 (gemeinschaftlich bearbeitet mit F. Seidl) 


\section{Zeitschriftenschlüssel}

Abh. aus d. Gesamtgebiet

d. Hygiene

Acta Phys. Austriaca

Almanach d. Wr. Akad.

d. Wiss.

Ann. d. Phys.

Anzeiger d. Öst. Akad. d.

Wiss., mathem.-naturw. Kl.

Anzeiger d. Öst. Akad.

d. Wiss., phil.-hist. Kl.

Badgasteiner Badeblatt

Ber. d. Dt.

chem. Gesellschaft

Forsch. u. Fortsch.

Ion

Jahrb. d. Rad. u. Elektr.

Kultur d. Gegenwart

Medizin. Welt

MIR

Nature

Naturwissenschaften

Neues Wr. Tagblatt

Österr. Chemiker-Zeitung

Österr. Rundschau

Pharmazeut. Presse

Phys. Zts.

Rad. in Biologie und Heilkunde

Schola Hornana

Strahlentherapie

Umschau
Abhandlungen aus dem Gesamtgebiet der Hygiene (Hrsg. von R. Grassberger)

Acta Physica Austriaca

Almanach der Wiener Akademie der Wissenschaften

Annalen der Physik

Anzeiger der Österreichischen Akademie der Wissenschaften, mathematisch-naturwissenschaftliche Klasse (ab 1947)

Anzeiger der Österreichischen Akademie der Wissenschaften, philosophisch-historische

Klasse (ab 1947)

Badgasteiner Badeblatt.

Kurkommission Badgastein

Berichte der Deutschen chemischen Gesellschaft (Berliner chemische Berichte)

Forschungen und Fortschritte

Ion. A Journal of Electronics, Atomistics, Ionology, Radioactivity and Radiochemistry. London Jahrbuch der Radioaktivität und Elektronik Die Kultur der Gegenwart (Hrsg. von P. Hinneberg) Physik (unter Redaktion von E. Warburg), B. G. Teubner, Berlin und Leipzig

Die Medizinische Welt. Ärztliche Wochenschrift. Berlin

Mitteilungen aus dem Institut für Radiumforschung (1911-1926)

Mitteilungen des Instituts für Radiumforschung (ab 1927)

(Sonderabdrucke aus den Sitzungsberichten der Akademie der Wissenschaften, mathem.naturw. Klasse, Abt. IIa.)

Nature

Die Naturwissenschaften

Neues Wiener Tagblatt

Österreichische Chemiker-Zeitung, Wien

Österreichische Rundschau, Wien

Pharmazeutische Presse

Physikalische Zeitschrift

Radium in Biologie und Heilkunde

Schola Hornana, Wien

Strahlentherapie, Berlin

Die Umschau. Wochenschrift über die Fortschritte in Wissenschaft und Technik. (Hrsg. von J. H. Bechhold) Frankfurt a. M. 
Urania. Illustrierte populärwissenschaftliche Wochenschrift. Offizielles Organ des Volksbildungs-Institutes „Wiener Urania“, Wien

Ver. z. Verbreitung naturw. Verein zur Verbreitung naturwissenschaftlicher Kennt. Kenntnisse in Wien

Verh. d. Dt. Phys. Ges. Verhandlungen der Deutschen Physikalischen Gesellschaft

Verh. d. Ges. Dt. Naturf. Verhandlungen der Gesellschaft Deutscher und Ärzte Naturforscher und Ärzte.

Vierteljahresbericht d. Wr. Vierteljahresbericht des Wiener Vereins zur Ver. z. Förd. d. phys. u. chem. Förderung des physikalischen und

Unterr. chemischen Unterrichts, Wien

Wiedemann Ann. d. Phys. Annalen der Physik

u. Chem.

Wiener Anzeiger

Wiener Sitz. ber.

(Akademischer) Anzeiger der Kaiserlichen Akademie der Wissenschaften in Wien (bis 1918) Anzeiger der Akademie der Wissenschaften in Wien (ab 1918)

Anzeiger der Österreichischen Akademie der Wissenschaften.

mathematisch-naturwissenschaftliche

Klasse (ab 1947)

Sitzungsberichte der kaiserlichen Akademie der Wissenschaften in Wien. Mathematisch-naturwissenschaftliche Classe; Band • Abteilung IIa <Monat Jahr> (bis 1915)

Sitzungsberichte der Kaiserlichen Akademie der Wissenschaften in Wien, Mathematisch-naturwissenschaftliche Klasse, Abteilung IIa, • .

Band, • . Heft (1915-1918)

Sitzungsberichte der Akademie der Wissenschaften in Wien, Mathematisch-naturwissenschaftliche Klasse, Abteilung IIa, •. Band, • . Heft (1918-1948)

Sitzungsberichte der Österreichischen Akademie der Wissenschaften (ab 1948)

Wissen f. Alle Das Wissen für Alle

Wr. Ztg.

Wiener Zeitung

Wr. Med. Wochenschr.

Wiener Medizinische Wochenschrift

Zeitschrift für Naturforschung

Zts. f. Naturforsch.

Zeitschrift für Balneologie, Klimato- u. Kurorthygienelogie und Kurorthygiene

Zeitschrift für Elektrochemie

Zeitschrift für Instrumentenkunde

Zeitschrift für physikalische Chemie

Vorgelegt von w. M. WeRnER WeLZIG

in der Sitzung am 6. Oktober 2000. 
